\title{
«Li sens conmence contreval a filer...». Imaginaire du sang et hétérodoxies épiques dans La Bataille Loquifer
}

Carlos F. Clamote Carreto

\section{(2) OpenEdition}

Journals

Édition électronique

URL : http://journals.openedition.org/medievalista/217

DOI : 10.4000/medievalista.217

ISSN : 1646-740X

Éditeur

Instituto de Estudos Medievais - FCSH-UNL

Référence électronique

Carlos F. Clamote Carreto, « «Li sens conmence contreval a filer...». Imaginaire du sang et hétérodoxies épiques dans La Bataille Loquifer », Medievalista [En ligne], 10 | 2011, mis en ligne le 01 juillet 2011, consulté le 02 mai 2019. URL : http://journals.openedition.org/medievalista/217 ; DOI : 10.4000/ medievalista. 217 
Título: «Li sens conmence contreval a filer...». Imaginaire du sang et hétérodoxies épiques dans La Bataille Loquifer.

Autor(es): Carlos F. Clamote Carreto

Enquadramento Institucional: Universidade Aberta; Centro de Estudos sobre o Imaginário Literário (FCSH - UNL)

Contacto: ogmios@univ-ab.pt

Fonte: Medievalista [Em linha]. №10, (Julho 2011). Direc. José Mattoso. Lisboa: IEM.

Disponível em: http://www2.fcsh.unl.pt/iem/medievalista/

ISSN: 1646-740X

\section{Resumo}

O sangue é, por excelência, a substância vital e mortífera da qual se alimenta a escrita épica. Polissémico por natureza e reenviando a variados estratos culturais e simbólicos, este fluido tanto pode representar o princípio da continuidade ligado à memória genealógica como o sangue impuro que corre nas veias da linhagem maldita dos pagãos ou dos traidores. Marcado por interditos ancestrais que fazem estremecer o discurso (visão do sangue feminino) ou designando uma ferida que ameaça a integridade do herói épico transformado em vítima sacrificial cujo sangue, derramado sobre o campo de batalha, purifica e regenera o espaço reconquistado pelo logos cristão, o sangue recria e transmite um imaginário paradoxal no qual se projecta simultaneamente o devir da linguagem e o da própria canção de gesta. Daí se tornar tão frequentemente, em francês antigo, hipóstase do sentido (o sens) através de uma homofonia que sela os destinos de ambas as substâncias e engendra uma singular poética do sangue que procuraremos perscrutar através de um atípico poema épico composto entre finais do século XII e inícios do século XIII, La Bataille Loquifer.

Palavras-chave: Sangue, La Bataille Loquifer, simbolismo, poesia épica. 


\section{Abstract}

Blood is, by nature, the vital and deadly substance which feeds the Chanson de Geste. Gathering a large range of symbolic and cultural strata, this polysemic fluid can either serve to embody the principle of continuity of the genealogical memory or to serve as the conveyor of the impure blood that runs through the veins of the cursed lineages of pagans or traitors. Tainted by the mark of ancestral taboos that shake the poetic discourse ( as with the vision of the blood that women shed, for example) or assigning the dangers of an open wound which threatens the integrity of the epic hero transformed into a sacrificial victim whose blood, shed on the battlefield, purifies and regenerates the space conquered by the Christian logos, blood creates and transmits a paradoxical imaginary which is both projected and reflected in the lineage and in the language, which reflects in the epic poetry itself. Hence the fact that in Old French, blood becomes an hypostasis of sens (meaning) by means of a marvelous homophony which seals as one the common fortune of both the substances and determines the particularly striking and unique poetics of blood which we can detect in the study and analysis of an atypical epic poem composed somewhere between the end of the $12^{\text {th }}$ century and the beginnings of the $13^{\text {th }}$ century, La Bataille Loquifer.

Keywords: Blood, La Bataille Loquifer, symbolism, epic poetry. 


\title{
"Li sens conmence contreval a filer...". Imaginaire du sang et hétérodoxies épiques dans La Bataille Loquifer
}

\author{
Carlos F. Clamote Carreto
}

\begin{abstract}
Duodecima uero generatione acceperunt homines praeceptum dei ne sanguinem degustarent propter hoc enim diluium factum est.
\end{abstract}

Vincent de Beauvais, Speculum historiale, I, $100^{1}$.

D’un côté, l'écriture hémorragique réduit l'autre (tout autre) au silence [...]. Poussé à sa limite, cette économie menace le texte lui-même de disparition [...]. De l'autre côté, [...] la geste s'arrange toujours pour qu'une fois le père mort, il y ait toujours un fils, un petit-fils, un oncle ou un neveu qui relève le défi et relance ainsi le feude [...]. La transmission du souffle et du sang du père mort deviennent dès lors des figures du renouvellement de l'écriture, correspondant trait pour trait à l'une des figures centrales de la Poetria nova, au titre explicite, de Geoffroi de Vinsauf: la rejuvenatio des anciens topoi par une écriture nouvelle.

Alexandre Leupin, «Raoul de Cambrai: la bâtardise de l'écriture», p. 100-101².

\footnotetext{
${ }^{1}$ Cité par TARAYRE, M - «Le sang dans le Speculum Maius de Vincent de Beauvais. De la science aux miracula», in FAURE, M. - Le Sang au Moyen Âge. Actes du quatrième colloque international de Montpellier, 27-29 novembre 1997). Les Cahiers du C.R.I.S.I.M.A., 4. Montpellier : Presses de l’Université Paul Valéry, 1999, p. 351.
} 


\section{L’aménorrhée épique ou l'écriture infinie}

Si le sang est toujours un signe ambivalent qui tout à la fois repousse et séduit, répugne et exerce une irrésistible fascination en activant les schèmes et archétypes de l'imaginaires liés aux pulsions élémentaires de vie et de mort ${ }^{3}$, il l'est d'autant plus dans une civilisation comme celle du Moyen Âge qui commence, surtout à partir du XII ${ }^{\mathrm{e}}$ siècle, à s'interroger systématiquement sur l'énigme de la transsubstantiation au sein du rituel eucharistique ${ }^{4}$. Or cette question, qui rappelle que le Christianisme est une religion du sang sacrificiel violement déversé et précieusement recueilli (aspect sur lequel se fonde et se développe notamment la mythologie du Graal) qui elle-même s'ancre, ne serait-ce que par le biais de la différence et de la réécriture, sur un sacrifice fondateur dont le sang est toutefois étanché avant-même qu'il ne jaillisse (Abraham), est peut-être avant tout une question d'ordre herméneutique: comment un corps physique donné change-t-il de nature et se sens? Comment écarter le spectre diabolique et terrifiant lié à la consommation du sang humain pour en faire un aliment régénérateur? Comment le sang visible qui jaillit d'un corps mourant devient-il promesse de rédemption et de vie éternelle? Comme on pourra le deviner, sous toutes ces interrogations s'inscrit la hantise du spectre gnostique du corps purement spirituelle, désincarné et d'essence fantasmagorique ou fictionnelle ${ }^{5}$, tout comme s'inscrit également la tension (parfois conflictuelle) entre le statut littéral, corporel et tangible du sang et sa nature métaphorique et figurée; une tension qui parcourt et structure la plupart

\footnotetext{
${ }^{2}$ Romanic Review, 79 (1988), p. 89-104.

${ }^{3}$ Voir, à ce sujet, l'étude de J.-P. Roux qui scrute les diverses (mais bien souvent convergentes) significations/utilisations et croyances sur le sang dans différents contextes culturels et historiques: Le Sang. Mythes, symboles et réalités. Paris : Fayard, 1988.

${ }^{4}$ Voir RUBIN, M. - Corpus Christi: The Eucharist in the Late Medieval Culture. Cambridge: Cambridge University Press, 1991; PALAZZO, E. - Liturgie et société au Moyen Âge. Paris: Aubier, 2000; MCCRACKEN, P. - «The Grail and Its Hosts», in The Curse of Eve, The Wound of the Hero. Blood, Gender, and Medieval Literature. Philadelphia: University of Pennsylvania Press, 2003, p. 92-117).

${ }^{5}$ Sur cette question, je renvoie aux arguments habilement mis en œuvre par Tertullien, notamment dans le traité De Carne Christi ainsi qu'aux réflexions d'A. Leupin dans Fiction et Incarnation. Littérature et théologie au Moyen Age. Paris : Flammarion, 1993.
} 
des récits et qui désigne en fait le paradoxe constant entre l'enveloppe mortelle et idolâtre de la littera et le caractère vivifiant de l'esprit qui, en la déchirant, la féconde ${ }^{6}$.

De ce paradoxe premier découle, en quelque sorte, tous les autres, le sang devenant, comme le soulignent Jacques Le Goff et Nicolas Truong ${ }^{7}$, véritable «pierre de touche» des rapports sociaux au cœur d'un Moyen Âge qui célèbre, autour de ses guerriers et du panthéon littéraire érigé par les chansons de geste, le sang déversé par fidèles et païens au nom de l'intégrité du logos chrétien, en même temps qu'il interdit aux oratores de le répandre et prescrit aux chevaliers un temps pour la guerre et un temps pour la paix; qui prohibe le sang impure versé par certaines professions ${ }^{8}$ au même titre qu'il exalte le sang des martyrs; qui manifeste ouvertement sa répugnance envers tous ces fluides corporels (surtout lorsqu'ils émanent du corps féminin) en même temps qu'il fait du sang l'élément central autour duquel se structure l'imaginaire généalogique 9 . Au carrefour, loin d'être pacifique, du discours médical (avec, en premier lieu, les apports de Galien, puis ceux d'Aristote, développés dans le cadre de l'École de Salerne) ${ }^{10}$, théologique et philosophique, le Moyen Âge semble redécouvrir le sang, comme origine et fin, toutes deux pareillement ambivalentes, de toute chose. Dans le registre assez virulent qui le caractérise, le De Miseria condicionis humane de Lothaire de Segni (futur Innocent III) s'inaugure sur une vision terrifiante du genre humain: souillé par les fluides impures - sperme et sang $^{11}$ - qui habite le ventre maternel, véritable

\footnotetext{
${ }^{6}$ Enjeu central de l'herméneutique chrétienne suggéré par le célèbre anathème lancé par saint Paul aux Corinthiens (II, 3, 6) - «Littera occidit, spiritus autem vivificat» - et amplement repris par saint Augustin dans le De Doctrina Christiana (III, V, 9 sq.).

${ }^{7}$ Une histoire du corps au Moyen Âge. Paris : Liana Levi, 2003, p. 39.

${ }^{8}$ Voir LE GOFF, J. - «Métiers licites et métiers illicites dans l'Occident médiéval», in Pour une autre Moyen Âge. Temps, travail et culture en Occident. Paris : Gallimard, 1977, p. 91-107.

${ }^{9}$ Voir VAN PROYEN, M. - «Sang et hérédité. À la croisée des imaginaires médicaux et sociaux des XIII ${ }^{\mathrm{e}}$ et $\mathrm{XIV}^{\mathrm{e}}$ siècles», in Le sang au Moyen Âge, p. 69-75.

${ }^{10}$ Pour les sources et les principales théories sur le sang (notamment sur les suspicions concernant sur le sang et les fluides féminin) dans le cadre de la médecine médiévale, voir JACQUARD. D. et THOMASSET, Cl. - Sexualité et savoir médical au Moyen Âge. Paris : PUF, 1985 ; LE GOFF, J. et TRUONG, N. - Une histoire du corps, p. 119-121), ainsi que l'excellente synthèse de THOMASSET, Cl.«De la nature féminine», in KLAPISCH-ZUBEL, Ch. (dir.) - Histoire des femmes en Occident. II - Le Moyen Âge. Paris : Plon, 2002, p. 65-98.

11 À la suite des auteurs de l'Antiquité, dont la pensée s'enrichit peu à peu grâce aux apports des médecins et philosophes arabes, le Moyen Âge reprend et développe la théorie selon laquelle tous les fluides, plus ou moins impurs ou inquiétants selon les contextes, dérivent du sang, qu'il s'agisse du sperme, de la sueur, de l'urine ou du lait.
} 
préfiguration du tombeau qui attend l'homme au moment de sa mort (de utero translatus ad tumultum, I, $1^{12}$ ), engendré dans l'angoisse et la douleur, l'homme est, dès l'origine, marqué par la condition humaine de pécheur, une condition inscrite dans la matrice même de la mère (cette éternelle Ève) dont le sang menstruel, fluide venimeux et létal par excellence, émerge comme miroir négatif de la Création. Cependant, Lothaire surenchérit encore sur cette vaste tradition théologique et scientifique, en faisant des premières voyelles à peine prononcées par le nouveau-né, non pas une parole adamique témoignant de l'innocence et de la pureté primordiales, mais une grammaire balbutiante de la Chute qui marquera à jamais le langage du sceau de la faute et de l'imperfection:

Sed attende quo cibo conceptus nutriatur in utero: profecto sanaguine menstruo, qui cessat ex femina post conceptum ut eo conceptus nutriatur in femina. Qui fertur esse tam detestabilis et immundus ut ex eius contactu fruges non germinent, arescant arbusta, moriantur herbe, amittant arbores fetus; si canes inde commederint, in rabie, efferantur (De miseria condicionis, I, 4).

Omnes nascimur eiulantes ut nature miseriam exprimamus. Masculus enim recenter natus dicit «A», femina, «E». Dicentes «A»vel «E» quotquot nascuntur ab Eva (De miseria, I, 6).

Comme partout ailleurs aux $\mathrm{XII}^{\mathrm{e}}$ et $\mathrm{XIII}^{\mathrm{e}}$ siècles, cet exemple montre clairement que le savoir livresque prédomine sur l'expérience empirique et que, à l'instar du regard porté sur le corps, le sang est un signe fait pour être lu avant d'être matière organique qui s'offre à l'analyse ${ }^{13}$. Il relève par conséquent tout autant (ou davantage encore) de la

\footnotetext{
${ }^{12}$ Éd. R. E. Lewis : Athènes, 1978.

${ }^{13}$ Comme le rappellent J. LE GOFF et N. TRUONG (Une histoire du corps, p. 131), l'ouverture des corps était davantage destinée à confirmer les théories de Galien plutôt qu'à chercher de nouvelles interprétations pour les phénomènes médicaux.
} 
sémiologie et de l'art de l'exégèse que de la science médicale. Le roman courtois ne manquera pas d'exploiter l'énorme pouvoir de représentation de cette image à la fois suggestive et extrêmement plastique qui devient ainsi un signifiant privilégié de l'écriture du désir ${ }^{14}$. Interpréter le discours polysémique du sang n'est toutefois jamais un exercice aisé. Que l'on prenne à témoin Tristan (dans la version de Béroul) dont la blessure s'ouvre juste au moment où il croyait éviter le piège tendu par le nain Frocin dont le but était de voir finalement s'inscrire sur la fleur de farine répandu entre les lits les signes tangibles (la «veraie enseigne», v. $778^{15}$ ) de la relation coupable. Ce qu'il réussit à prouver, bien que les signifiants constitutifs de cette singulière écriture finissent par échapper à son projet initial ${ }^{16}$. Que l'on songe également au scénario plus complexe développé par Chrétien de Troyes dans Le Chevalier de la charrete où l'accès à la femme interdite engendre une blessure sanglante qui tâche les draps de Guenièvre, mais dont personne (ni même les amants) n'en connait ni l'origine, ni le sens, ni même les implications profondes. L' «ensaignes bien veraie» (v. 4774) ${ }^{17}$ dit alors tout moins la vérité de l'adultère, dénonçant, malgré l'invention extrêmement symptomatique de la reine qui imagine avoir saigné du né ${ }^{18}$, un Keu qui, pour une fois, était pourtant innocent. Signe extrêmement ambigu et redoutable, le sang est ainsi surtout une image-

\footnotetext{
14 Attirant au point de pétrifier le héros au seuil d'une l'extase mortelle, ravissant et aux effets potentiellement dévastateurs, nous trouvons le discours du sang au centre du parcours de Perceval de Chrétien de Troyes lors du célèbre épisode des trois gouttes de sang sur la neige où l'oubli amoureux du valet gallois émerge sur fond d'une violente et sanglante étreinte sexuelle marqué par la faille et l'impossibilité d'atteindre/de saisir l'autre dans da plénitude. Le sang répandu par l'oie sauvage puis gommé de cette surface neigeuse devenue emblème du palimpseste, n'est ainsi que l'image déplacée de ce drame infiniment rejoué autour d'un manque à dire primordial qui se place entièrement sous le signe de la blessure et du sang, le silence du nice sur le cortège du Graal (qui contient l'aliment eucharistique, comme nous le saurons par la suite) et la Lance-qui-Saigne empêchant la régénération de cet autre-monde dont la stérilité mortelle est justement emblématisée par l'infirmité du Roi Pêcheur blessé - comme le fut naguère le père du héros - entre les jambes. Dans les deux cas, la faute de Perceval n'est pas tant dans le silence, infécond en soi, mais plutôt dans le fait qu'il s'agisse-là d'un silence qui refuse (consciemment ou non) d'interroger un rituel qui a un rapport singulier au sang à travers l'émergence d'une parole qui permettrait d'en circonscrire/saisir le sens et, par conséquent, de stopper cette énigmatique hémorragie qui finira par affecter, à la fin du récit, le royaume même d'Arthur.

${ }^{15}$ BÉROUL, Le roman de Tristan, éd. LACROIX, D. et WALTER, Ph. - Tristan et Iseut: les poèmes français, la saga norroise. Paris : Librairie Générale Française, Coll. Lettres Gothiques, 1989, p.21-231.

${ }^{16}$ Voir notamment les réflexions de HUCHET, J.-Ch. - Tristan et le sang de l'écriture. Paris : PUF, 1990.

${ }^{17}$ Éd. ROQUES, M. Paris : Champion, 1983.

${ }^{18}$ Ce qui dévoilerait, sous le signe du mensonge ou du voile fictionnel, un autre type de vérité inconsciente (l'interdit sexuel durant la menstruation). Avant que la théorie psychanalytique n'ait fait le rapprochement entre le saignement nasal et le sang menstruel (voir, à ce sujet, MCCRACKEN, P. - The Curse of Eve, p. 13-14), déjà Aristote, suivi par la pensée médiévale que si les femmes n'ont pas de saignement de né, c'est parce qu'à défaut de pouvoir se purger de leurs résidus par la chaleur du corps, elles se purifient régulièrement à travers les menstrues (THOMASSET, Cl. - «De la nature féminine», p. $80)$.
} 
écran qui occulte plus qu'elle ne dévoile. Pourquoi Méléagant et son père refusent-ils de croire aux paroles de Guenièvre? Pourquoi ce sang répandu sur les draps ne peut-il pas appartenir à la femme? Voici qui nous relance directement dans la problématique, apparemment plus linéaire, du sang épique.

En effet, la fiction de Chrétien révèle que le sang appartient avant tout au langage masculin, et que, dans cette perspective, il ne peut jamais (ou rarement) pointer vers l'intime troublant d'une histoire centrée sur le corps féminin, désignant plutôt cette tension/lutte aux contours anthropologiques entre les hommes pour la possession de la femme, et, à travers elle, de la terre, du nom, etc. À l'exception des vierges vouées au $\operatorname{martyr}^{19}$, les femmes ne saignent pas, ou presque pas, dans la littérature, le sang féminin (plus que tout autre fluide corporel) étant un élément anxiogène qui, comme l'ont parfaitement montré les récentes études de Peggy McCracken ${ }^{20}$ et de Finn E. Sinclair ${ }^{21}$, représente toujours une puissante menace pour l'ordre symbolique et textuel qu'il faut à tout prix chercher à gommer au nom de l'intégrité d'un logos calqué sur le modèle masculin et patricial. La chanson de geste se constitue à partir de cette antinomie discrète bien que structurante. Car, à l'inverse des femmes et du tabou sur le sang qui les stigmatise dès le début des temps, l'ethos guerrier se définit, au contraire, par le don du sang. Comme on peut s'en douter à en croire cette «poétique du génocide joyeux» ${ }^{22}$ qui caractérise le discours épique dès ses origines scripturaires, le sang est un élément consubstantiel à l'épopée inévitablement vouée, en ce sens, à l'aporie. Que la menace vienne de l'intérieur de la Chrétienté (motif de la trahison, cycle des barons révoltés) ou qu'elle se projette sur l'Autre par excellence représenté par les païens, il y aura toujours un sang à venger et à étancher, comme le montre exemplairement le cycle infernal de la violence mis en œuvre dans Raoul de Cambrai. Et c'est pourquoi je suis persuadé que la constitution en cycles (surtout à partir du XIII ${ }^{\mathrm{e}}$ siècle) était en quelque sorte le devenir logique et incontournable de l'écriture épique, car nul mieux que l'extension cyclique et

\footnotetext{
${ }^{19}$ PERROT, J.-P. - «Du sang au lait: l'imaginaire du sang et ses logiques dans les Passions de martyrs», in Le Sang au Moyen Âge, p. 459-470.

${ }^{20}$ The Curse of Eve.

${ }^{21}$ Milk and Blood. Gender and Genealogy in the «Chanson de Geste». Berne: Peter Lang, 2003.

${ }^{22}$ PAYEN, J.-Ch. - «Une poétique du génocide joyeux: de voir de violence et plaisir de tuer dans la Chanson de Roland», Olifant, 6, 3-4 (1979), p. 226-236.
} 
son triple mouvement basé sur l'effacement, l'amplification ou la réécriture, ne saurait exprimer la logique oblative et spiroïdale qui gouverne l'imaginaire du sang épique: un sang répandu qu'il faut constamment racheter en faisant couler un nouveau sang ou qu'il faut chercher à stopper (lorsqu'il s'agit d'éviter une mort qui menace de faire s'effondrer l'ordre idéologique et symbolique) en reconstruisant l'image d'un corps intègre. Voilà qui relève d'une premier paradoxe: le fait que le sang coule en abondance n'ôte en rien sa nature angoissante, surtout, bien entendu, lorsqu'il jaillit du corps du héros chrétien. Cette hantise du corps décomposé, démembré, du corps introuvable, du corps désincarné qui ne peut plus faire l'objet d'une commémoration, interdisant, par conséquent, l'émergence du chant épique qu'il met totalement en déroute, est exemplairement mise en scène dans la première partie de la Chanson de Guillaume qui offre le spectacle tragique d'un univers idéologique et textuel en décomposition à travers la mort silencieuse, solitaire et sans aucune gloire de Vivien ${ }^{23}$. Comment ressouder alors le corps? Comment stopper l'hémorragie si l'écriture épique est par définition une plaie mal soignée toujours prête à se rouvrir, une blessure qui en appel toujours une autre et dont le sang et le sens s'écoulent sans cesse sur un nouveau récit? La désincorporation épique est en réalité toujours appel discret à la pratique de la continuation et de la récriture édifiée sur la glose et la réinterprétation constante du corps. Parfois, il s'agira tout simplement de réinventer une mémoire, la trouvaille poétique consistant à retrouver et à recomposer le corps perdu. Le texte assume alors les contours d'une écriture que l'on greffe sur une forme antérieure dont le statut ambigu l'empêchait de s'ériger en modèle valable pour la chanson de geste à venir. C'est ce que l'on observe dans la seconde partie de la Chanson de Guillaume et dans Aliscans qui se

\footnotetext{
${ }^{23}$ En effet, resté seul sur le fatidique lieu de Larchamp (espace où résonne encore, sur le plan de l'imaginaire, la défaite traumatisante de Roncevaux) où les troupes françaises sont décimées, le héros se désincorpore progressivement de tous ses attributs guerriers. Après la mort de son cheval, il souffre les effets de la faim, de la soif, de la chaleur insupportable et de la fatigue physique et existentielle. Ayant semé stérilement sur le chemin ses armes, symbole de la dispersion d'un Chrétienté en péril et d'une désintégration de la sémiologie épique, il laisse finalement tomber l'emblème ultime de l'identité, l'étendard (laisses 64-65 de l'éd. de REGNIER, Cl. Paris : Champion, 2 vols, 1990). Les innombrables blessures infligées à son corps transforment celui-ci en un objet poreux (laisses 65-71) qui se vide peu à peu de sa substance vitale (sang, organes, eau). La mort approchant, les yeux «li sunt trublez» et même le désir de revoir une dernière fois Guillaume lui est refusé (v. 895). Dilacéré par les païens afin qu'il ne puisse être retrouvé, son corps est fragmenté («Tuit le detrenchent contreval al graver», v. 925) et dissimulé. Placé sous le signe de la dispersion, la mort de Vivien n'a rien d'un sacrifice régénérateur (modèle rolandien) et la désincorporation du héros, touchant à l'effacement, conduit l'écriture épique à l'impasse devant l'absence de tous signes corporels à partir desquels on puisse lire/écrire les vestiges d'une geste exemplaire et structurer une mémoire fondatrice/identificatrice.
} 
proposent de réécrire la mort de Vivien en la réajustant au modèle christique et rolandien du héros mourant dont le corps, désormais entier et serein, est trouvé encore en vie en plein un locus amoenus paisible par Guillaume qui confesse et donne la communion au héros avant de le transporter à Orange où il sera dignement enterré et commémoré. La transformation est révélatrice, dans la mesure où elle met clairement en scène une idéologie et un imaginaire du corps masculin qui représente l'envers (ou le négatif) du discours sur le corps féminin: l'idéal du corps intègre, étanche, non corrompu (même dans la mort ou surtout dans la mort), parfaitement maîtrisé et qui maintient intacte l'empreinte de la figura moulée par Dieu, face à l'image d'un corps totalement perméable et fluide, incohérent, incontrôlé, mystérieux et menaçant ${ }^{24}$. Comme l'affirme Peggy McCracken,

The prominent representation of men's blood in battles also revises the characterization of gendered bodies in medical and theological discourses, where the idealizes male body is usually described as sealed, intact and conservative; by contrast, the female body is unruly, uncontained, and permeable ${ }^{25}$.

Cette représentation du corps correspond évidemment à l'imaginaire généalogique et discursif de la chanson de geste ancré dans les principes de continuité, de permanence et de linéarité, comme l'a exemplairement démontré Howard Bloch ${ }^{26}$, qui décrivent un univers où le fils est la représentation métonymique du père, ce dernier pouvant donc disposer de la vie de son descendant qui se sacrifie au nom de la pérennité du lignage et du langage. Rien d'étonnant donc à ce que la geste soit entièrement parcourue par la

\footnotetext{
${ }^{24}$ Comme le rappelle F. E. SINCLAIR, c'est justement parce que le corps féminin est un corps poreux, perméable d'où s'écoulent des fluides énigmatiques tenues comme potentiellement néfastes qu'il devient figure de l'altérité par excellence: «The construction, presentation, and manipulation of the female character in the text reflects this predominance, yet, $[\ldots]$ the conflicting impulses within contemporary ideaological discourse can work to produce na aver-all image of women which is incoherent or fragmebted [...]. The definition of this space [le corps maternel] is to great extend predicated on the alterity and specificity os the female body: a body that bleeds, secretes, gestates and lactates» (Milk and Blood, p. 19).

${ }^{25}$ The Curse of Eve, p. 14.

${ }^{26}$ Étymologie et généalogie. Une anthropologie littéraire du Moyen Age français. Paris : Seuil, 1989, p. 113-145.
} 
hantise de l'interruption généalogique, de la rupture ou de toute dérive métaphorique qui mettrait en cause l'idéal de la proprietas (au sens généalogique, linguistique ou économique): l'intégrité du corps masculin épique n'est donc que la manifestation tangible d'un système idéologique et poétique qui prône la cohésion, la transparence et la perméabilité des signes (de tout signe) dans leur rapport à un Signifié transcendant, stable et toujours disponible. À l'opposé, le corps féminin représente l'émergence troublante et déstructurante du désir. C'est ce que montre à plusieurs reprises le poème sur lequel viendront s'appuyer nos réflexions, La Bataille Loquifer, où la vengeance des Sarrasins à l'égard des héros chrétiens (avec Rainouart et Guillaume d'Orange au premier plan) commence toujours par prendre la forme d'une irrésistible violence (physique et verbale) exercée sur la femme. Destiné à circuler entre les hommes comme signifiant privilégié de l'échange oblatif qui structure l'économie idéologique, symbolique et narrative de la chanson de geste, le corps féminin semble ne pouvoir être touché, saisi, compris et communiqué qu'à travers le langage masculin de la déchirure. Tout d'abord à l'égard d'Aélis, la femme de Rainouart, dans un passage dominé par une rhétorique du non-dit (ou de l'indicible) d'où émerge assez clairement toutefois le spectre du viol et de la sexualité contre-nature:

«Et de sa feme ferons nos volentés» (v. 129) ${ }^{27}$

«Sa feme avront garçon et pautonnier.»

La dame prisent et devent et daier,

Tot li desirent son fres hermine chier (v. 260-262)

Remarquons, dans ce passage, le subtil déplacement sémantique bâti sur le rapport métonymique entre la parure claire et précieuse d'hermine et la peau blanche d'Aélis, ainsi que sur le jeu autorisé de la dubia locutio autour de la forme verbale «desirent» qui renvoie aussi bien à la déchirure (des vêtements et du corps) sous l'effet la violence

${ }^{27}$ Éd. BARRETT, M. Meddium Aevum Monographs, New Series, 6: Oxford, Society for the Study of Mediaeval Languages and Literatures, 1975. 
exercée sur la femme qu'à la dimension même du désir. Même processus rhétorique un peu plus tard dans le texte lorsque, profitant de la bataille générale qui retient Guillaume d'Orange et Rainouart sur l'île où a eu lieu le combat contre le géant sarrasin Loquifer (nous y reviendrons), le roi païen Thibaud capture Guiborc (alias Orable, la princesse sarrasine qui fut naguère sa femme avant de devenir celle de Guillaume et de se convertir au Christianisme dans la Prise d'Orange) qu'il humilie, taxe de luxure et tire par les cheveux en la traînant par les rues de Porpaillart. Or, le comportement de Thibaud, qui n'a jamais compris pourquoi il a été délaissé et qui traduit à la fois un désir de vengeance et une irrésistible volonté de savoir, de percer le secret du féminin, se manifeste également à travers une expression doublement signifiante fondée sur l'homonymie du terme desirer. Le texte raconte, en effet, que Tibaut s'est acharné sur Guibourc au point «que sa robe en covint desirer» ([«que sa robe il en vint à déchirer», v. 2647). De la déchirure au désir, il n'y a donc qu'un pas, la déchirure du désir transformant la parure en haillon face à l'impossibilité de posséder le corps et de (trans)percer le secret qu'il abrite. Violement désiré, violé, agressé ou usurpé et déchiré, le corps de la femme n'est cependant jamais montré comme un corps sanglant, bien que l'image du sang (surtout celle du mauvais sang) y soit constamment présente à travers le spectre de la bâtardise. Dans La Bataille Loquifer, cette bâtardise renvoie, bien entendu, tout d'abord, au renoncement de Rainouart à la religion païenne. Cependant, inscrite dans la séquence du corps violé et à la suite d'une rhétorique de l'équivoque dominée par l'annominatio, ne pourrait-elle pas être également prise au sens littéral (biologique), lançant alors un doute sur la légitimité du statut paternel de Rainouart? Autre aspect remarquable qui renforce l'ambiguïté de ce scénario imaginaire et qui n'est sans doute pas, en ce sens, le fruit du hasard: les expressions à travers lesquelles le sarrasin Clarion manifeste au héros son désir de tuer Aélis et de mettre ainsi fin à sa descendance (contrariant ainsi l'estoire, i.e., la source écrite du récit, la réécriture païenne se plaçant ainsi sous le signe de la négation et de la contrefaction de la lettre primordiale, attitude qui est l'exacte envers d'une poétique chrétienne et épique ancrée dans le principe de la rectitude et de la fidélité aux noms et aux sources autorisées du conte, comme le suggère la rhétorique mis en œuvre dans de nombreux prologues de romans et de chanson de geste), reprennent termes pour termes ceux qui décriront la mort d'Aélis lors de l'accouchement de Maillefer, comme si les désirs pervers des païens prenaient soudain corps dans l'inter-dit de la fiction: 


\author{
«[...] a ta feme que tu as esposé \\ seront overt li flanc et les costé \\ pour lou bastart que aves engendré, \\ dont est ensainte bien a .VII. mois passé; \\ car nos l'avons en l'estore trové \\ que par ton filz serons deserité; \\ mais nostre livre en seront tuit falsé» (v. 416-422).
}

Tant travailla [dame Aëlis] ans que l'anfes fust nés, li cuers li crieve, ses cors est detiués; si li ovrirent les flans et les costés, l'anfent en traient qui fu gros et quarrés (v. 783-785).

Il est intéressant de noter que, bien qu'il souligne la violence de cette naissance sous fond de mort, le manuscrit 1448 de la Bibliothèque Nationale de France (qui sert de base à l'édition de Monica Barnett) gomme totalement l'image du sang répandu (sang à la fois impur et fondateur d'une nouvelle lignée de héros) qui devrait pourtant caractériser cette séquence. Palimpseste que l'amplificatio du ms E (de la Bibliothèque Municipale de Berne) nous permet toutefois de récupérer en mettant en scène l'image toujours ambigüe d'un fluide (s'agit-il du sang de la parturition, de la mort et/ou celui qui provient de la blessure que s'auto-inflige Rainouart?) qui, récupérant toute sa force expressive, recouvre désormais le visage et les mains de Rainouart déchiré par la douleur:

Morte est la dame qui tant est bele et fine [...].

Quant R. en oï la covine,

il tort ses puins et sa face gratine;

dou sang qui chiet devint toute sanguine $(783-785)^{28}$.

\footnotetext{
${ }^{28}$ Version donné par BARNETT (éd. cit. p. 150).
} 


\section{Une grammaire du paradoxe: le sang ou la dérive généalogique et textuelle}

La Bataille Loquifer semble d'ailleurs entièrement gouvernée par cette logique du gommage et du déplacement. Déplacement - et nous aurons l'occasion d'y revenir -, parce qu'elle se présente comme perpétuel mouvement entre le sens figuré le sens littéral, le récit étant en fait l'histoire d'une série de métaphores (ainsi que l'histoire des désirs qui les sous-tendent) qui progressivement acquièrent une étrange autonomie au sein du récit, finissant par incarner dans la fiction (ou sous forme de fiction). Quant au gommage, il affecte la rhétorique et l'imaginaire même du sang qui assume ainsi une présence/absence paradoxale dévoilant les contradictions ou les tensions poétiques et idéologiques sous-jacentes au récit. L'histoire racontée par cette singulière chanson de geste anonyme datant sans doute du début du XIII ${ }^{\mathrm{e}}$ siècle se résume en peux de mots: après une double séquence typiquement épique centrée sur la bataille qui oppose, d'une part, Rainouart au géant Loquifer et, d'autre part, Guillaume au roi païen Déramé (nous devinons-là une structure en forme de diptyque qui met en scène le combat décisif entre doubles mythiques et littéraires $)^{29}$, la chanson de geste, recourant au topos particulièrement fertile de la famille dispersée, se détourne de son registre poétique dominant pour entrer dans le Royaume de la Féerie. Ainsi, alors qu'il quitte Guillaume pour partir à la recherche de son fils prisonnier à Loquiferne, Rainouard, endormi sur la rive de Porpaillart, est soudain ravi par trois fées (dont Morgue et sa sœur Marsion) qui l'emportent en Avalon ${ }^{30}$ où il sera présenté à Arthur, Roland, Gauvain, Yvain, Perceval et Guenièvre (v. 3898-3908) qui ont fini leur vie et passent maintenant l'éternité en compagnie des fées. Le héros devra alors affronter, sur le commandement d'Arthur, Chapalu, une laide beste (v. 3750), durant un combat qui dissipera et le sortilège dont il fut victime (recouvrant alors sa figure humaine après avoir bu le sang du talon de Rainouart) et, à en croire les paroles énigmatique du roi, les enchantements de l'Autre-

\footnotetext{
${ }^{29}$ Cette séquence rend compte, en amont, d'un premier stratagème (manqué) des païens (déguisés en marchands) envoyés par Thibaut pour s'emparer de Rainouart et d'Aélis (laisses I-XI), l'objectif étant la reconquête d'Orange et d'Orable (comme si le Cycle de Guillaume d'Orange se déployait maintenant à rebours), et, en aval, de la naissance de Maillefer (qui entraîne la mort d'Aélis - laisse XI) et de son enlèvement par l'étrange Picolet lou legier, le nain au service du géant païen Loquifer et de Thibaut (laisse 48).

${ }^{30}$ Le thème de l'enlèvement, fondé sur le schéma structural de la famille séparé (conte-type PlacideEustache: AT 938) est un schéma abondamment exploré par la chanson de geste à partir du XIII ${ }^{\mathrm{e}}$ siècle permettant, au niveau structurel, de relier la partie épique du récit à la partie «romanesque» (Rainouart à Avalon).
} 
Monde, car, à la suite de cette victoire, Rainouart «l'ostel desraisnera» (v. 3789) à Chapalu, prenant alors possession du Royaume de Féerie. Une fois l'exorcisme opéré, Rainouart passe la nuit avec Morgue et conçoit le diabolique Corbon. Le retour d'Avalon est assez agité, car Chapalu (sur le conseil de Morgue) provoque un naufrage durant lequel le héros, gravement blessé et ne sachant pas nager, croit toucher à sa fin. Il sera cependant sauvé par les sirènes qui l'endorment de leurs chants et le ramènent exactement au point de départ, i.e., sur le rivage de Porpaillart où il se réveille endeuillé par la mémoire d'Aélis et la perte de Maillefer.

Comme beaucoup de poèmes du même genre, cette chanson de geste est entièrement dominée par le spectre du corps morcelé, déchiré, fragmenté (les scènes de bataille occupant près de 70 laisses sur 94). Le sang y coule donc abondamment, pourrait-on penser. Mais est-ce vraiment le cas? Ne faut-il pas distinguer ce qui appartient simplement à la sphère de la suggestion (de l'inter-dit) de toutes ces formes poétiques qui accèdent à un statut différent par le biais de la nomination? Une simple analyse lexicographique suffit à montrer que le nombre d'occurrences du mot sang (ou de ses dérivés proches) est en fait assez réduit vu le contexte narratif du poème: près de vingtquatre cas qu'il faut encore répartir selon deux catégories sémantiques différentes. Dans huit cas, le lexème est utilisé au sens figuré pour exprimer la rage, la colère et la démesure typiques de cette humeur sanguine ${ }^{31}$ qui est loin d'être une caractéristique propre du héros masculin et du furor épique qui s'empare de lui durant le combat:

Rainoars l'ot, pres n'a lou sanc desvé (v. 438).

\footnotetext{
${ }^{31}$ La complexion sanguine (chaude et humide) ne se traduit pas forcément en violence et en démesure irrationnelle, les vertus guerrières des héros sarrasins ainsi que leur respect méticuleux du code et de l'éthique chevaleresques étant clairement soulignés au long de La Bataille Loquifer. Elle se caractérise toutefois par une tendance à extérioriser les pulsions à travers un langage du corps qui se situe aux antipodes d'un certain idéal de contention: «Les sanguins qui ne prendent partie d'autre complexion si doivent estres [...] largues, amans, legiers, rians et vermaux, cantans, carnus, hardis par raison et debonnaires: tel doit estre nature li homs sanguins. Tele menniere de gent font volontiers coÿt et moult le peuent faire, pour ce qu'il sont cauls et moistes; par le caleur, il en sont talentieus; par le moisteur, il en peuent faire assés» (Placides et Timéo ou Li secrés as philosophes, 427. Éd. critique par THOMASSET, Cl. Genève : Droz, 1980).
} 
Li Sarrasins ot lou sanc esmeü (v. 1650).

Cant Renoars voit rote sa masue

Trestos li sens de fierté li remue (2333-34).

Desramé l'ot, s'a la color müe;

Li sens li fuit, s'ait la rene tiree (2728-29).

Morgue l'antend, lou sanc cuide desver (v. 3949) ${ }^{32}$

L'imaginaire lié au sang se laisse aisément dévoilé à travers les formes verbales qui côtoient le lexique sanguin: «esmouvoir», «remuer», «desver» évoquent le schème du mouvement énergétique et désignent l'aspect hyper-dynamique de ce sang qui coulent à l'intérieur des veines avec une telle ardeur qu'il menace à chaque instant de déchirer le corps, la pulsion de vie pouvant alors céder sa place à la mort, et le contrôle de soi à la démesure aux conséquences toujours imprévisibles par nature. Remarquons ainsi que ce sang n'est bienfaisant que dans la mesure où il demeure dans les limites strictes d'un corps parfaitement étanche. Lorsque le schème du mouvement (surtout lorsqu'il est mouvement désordonné) donne lieu à celui de l'épanchement et de la fuite, il y a toujours une partie de l'être qui risque de s'écouler avec le sang. Une fois de plus, le langage poétique saura tirer pleinement partie du jeu des signifiants, l'image éloquente du sang qui fuit, devenant, dans le vers 2729, hypostase du sens (la «raison») qui abandonne peu à peu le corps du roi Déramé alors qu'il sombre dans une espèce de folie guerrière. Bien qu'elle affecte potentiellement toute personne (homme ou femme), l'humeur sanguine semble surtout caractériser l'Autre, que celui-ci désigne ce nouveau champion de la Chrétienté qu'est Rainouart dont le corps continue néanmoins à exhiber les signes du gigantisme typiques du monde sarrasin dont il est originaire (deux

\footnotetext{
${ }^{32}$ Voir aussi le v. 1708 (colère de Loquifer), le v. 1502 (Loquifer arrachant «par maltalant» ses cheveux avec une telle rage «que li sens en est enprés saillis»: s'agissant d'un sang qui émerge de l'organe de la raison par excellence, le tête, l'écoulement du sang devient ici à nouveau clairement isomorphe d'une perte du sens), le v. 3486 (Thibaud apprenant la mort de Déramé) et le v. 3648 (rivalité entre les quatre fées à l'égard de Rainouart).
} 
occurrences), le guerrier païen, double parfait du héros carolingien auquel il manque seulement l'adhésion à la foi chrétienne (Déramé: deux occurrences; Loquifer et Thibaut: une occurrence chacun), ou encore la femme menaçante aux traits morganiens (les fées: deux occurrences). Le langage figuré du sang émerge ainsi comme discours idéologique permettant de construire/transmettre une certaine vision du monde et de l'ordre social et symbolique, La Bataille Loquifer dessinant, de façon subtile ou subliminaire, un univers où le vrai héros chrétien émerge, à l'image de Guillaume et à l'inverse d'un Roland ou d'un Raoul de Cambrai, comme modèle de la contention, d'un parfait contrôle sur soi, qui se projette sur l'image d'un sang dont le flux est pleinement maîtrisé à l'intérieur d'un corps étanche.

Toutes ces acceptions figurées du sang réduisent donc davantage encore les occurrences où ce fluide renvoie à une substance physique et qui se limitent au plus à 15 cas, tous situés dans le contexte épique de la bataille et concentrés surtout lors du duel aux contours eschatologiques entre Rainouart et Loquifer (8 occurrences) autour duquel se structure le récit. Dans tous ces emplois, ce n'est pas tant l'aspect dynamique du jaillissement que la dynamique verbale souligne, mais plutôt celle de l'écoulement. Ce fluide qui se répand à partir du corps blessé n'a rien du fluide impur qui caractérise, par exemple, le sang féminin dans les Passions de Martyr obligeant la rhétorique textuelle à métamorphoser ce liquide en un autre aux connotations plus positives (le lait, notamment). Déversé par les païens, il devient soit le sang qui alimente spirituellement (ou métaphoriquement) l'ennemi lui-même dont la force s'accroît par la vision de son propre sang épanché -

Li sens en cheit contreval an la pree;

voit lou li Turs [Déramé], s’ait la color müee, cant voit son sans, sa vertu est doublee.

«Glos», dist Guillelmes, «vostre vie est alee» (v. 3129-32) -, 
soit la manifestation d'un sacrifice fondateur qui purifie l'espace usurpé et souillé, tout en permettant au héros de la geste de recouvrer toute son énergie vitale qui se nourrit, elle aussi, de ce sang qui jaillit du corps de l'adversaire. Répandu par les chrétiens, il assume fréquemment les contours du sang christique versé pour le rachat de l'Humanité et le rétablissement de l'ordre social et symbolique, sans pour autant effacer le caractère terrifiant de cette vision du fluide vital qui abandonne fatidiquement, sans que rien ne puisse l'arrêter, le corps des héros sentant leur vie fuir à travers une chaire déchirée, transpercée, morcelée (les images sont assez expressives), une chair sur laquelle le guerrier n'a plus aucune maîtrise, qui a cessé d'être modèle d'intégrité, de cohésion et étanchéité:

Li sens conmence contreval a filer, ancontreval l'an veïssiés aler, il [Rainouart] n'a de coi puist sa plaie bander; se longues saine, ja ne pora durer (v. 1745-48).

Ans Loquifers ne daigna remüer,

Renoars voit, sel prent a avisser, lo sanc li voit encontreval filer, vers lui se torne, sel prent a apeler [...]:

«Dont vient cil sans que ge voi avaler, de tel randon sor cel hauber coler? [...] Desor ta char voi gel ou sanc beter (v. 1755-58; 1762-63; 1772-73).

Tote li a li brogne depanee et ça char route par delés l'eschinée; li sans an chiet a molt grant randonee (v. 2281-83).

En la char [Déramé] durement lou navra [Guillaume], si que li sans contreval en raia (v, 3402-03). 
Mais l'image, particulièrement vive, de ce sang qui tombe de toute sa pesanteur et qui s'écoule pour imprégner (et féconder?) la terre, la peau, les armes et la parure des guerriers, n'évoque pas uniquement un sang réparateur ou vengeur; elle revêt parfois les traits d'une singulière écriture, non seulement par les effets chromatiques qu'elle engendre, mais également grâce aux riches et constantes interférences/confluences sémantiques provoquées par la rencontre (voulue ou fortuite, mais toujours signifiante) entre le sang et le sens, unis au sein d'un jeu homophonique assez fréquent dans la littérature médiévale ainsi que tout au long de La Bataille Loquifer:

Li sans an cheit durement par lou pré (v. 1911).

Lor armeüre ont toute desronpue,

del sanc des cors est la terre vestue (v. 2000-2001).

Lou teste li froisse, la char est entamee [...];

[...] la en fut ansanglantee;

L'erbe en est roge, tainte et encoloree [...] (v. 2263; 2267-68).

Li cuens Bertrans n'en a nul esparnié, mais de l'ocire a tot lou branc sachié et bras et pons an sanc vermail baignié (v. 2868-70).

Del sanc vermail chascons ansanglanta (v. 3109).

Remarquons finalement que le lexique du sang n'est jamais convoqué lors du coup fatal qui tue Loquifer et Déramé, comme si l'unique sang qui intéressait véritablement l'imaginaire épique était le sang vif et chaud qui abreuve et régénère le héros, la terre et la geste, et non pas le sang noir et stérile de la mort. Le nombre réduit de lexèmes relatifs au sang biologique dans ses divers contextes sémantiques nous permet donc de conclure qu'il semble exister, tout au long de La Bataille Loquifer, une espèce de 
pudeur et de contention à l'égard de ce fluide. Peut-on y discerner la présence vestigiale d'un tabou archaïque sur le sang qui, malgré la surdétermination épique qui le définit comme offrande rituelle masculine, demeure un fluide dont la vision répugne et angoisse, dans la mesure où il évoque inévitablement l'image dévoratrice et destructrice du temps qui s'écoule, d'un univers qui se décompose et qui menace, à chaque instant, de s'interrompre, mettant fin au lignage et condamnant la mémoire et le chant épique qui la transmet et célèbre à l'effacement et au silence? Quoiqu'il en soit, ce poème traduit indiscutablement une tension à l'égard de l'image affichée du sang, fréquemment suggérée, mais souvent confinée au seuil d'une impossible nomination. Ce silence (relatif, bien entendu) ou cette contention dans le dire du sang serait-elle une forme se stopper l'hémorragie épique qui condamnait la chanson de geste, comme nous l'avons suggéré, à l'infinie (et presque rituelle) répétition d'une blessure qui, en se déplaçant de corps, d'espace, de temps et de récit, n'a cesse de se rouvrir? Cette hypothèse pourrait être étayée par la manière assez originale ${ }^{33}$ dont La Bataille Loquifer met en scène, par le biais d'un surprenant dialogisme avec la matière de Bretagne, le rapport entre le registre traditionnel de l'épopée et tous ces éléments narratifs exogènes à cette tradition. Le baume magique et guérisseur est, en effet, un des motifs structurant au long du récit qui prépare le basculement total (à la laisse 71) de la chanson de geste dans l'AutreMonde du roman arthurien ${ }^{34}$ et, par voie de conséquence, le déplacement de l'univers du symbole vers une vision du monde placée sous l'empire du signe ${ }^{35}$. Comme l'a

\footnotetext{
${ }^{33}$ Mais loin d'être unique au XIII ${ }^{\mathrm{e}}$ siècle: qu'on se souvienne de la pierre guérisseuse dans le Moniage Rainouart ou de l'herbe médicinale d'Orable (la princesse sarrasine qui prend remarquablement l'allure de la fée des récits bretons) dans les Enfances Guillaume. Cependant, l'organisation et l'omniprésence structurante de ces éléments hétérogènes à la tradition épique à l'intérieur du récit me semble assez singulière surtout dans un poème aussi bref que La Bataille Loquifer.

${ }^{34}$ Les indices sont nombreux tout au long du récit, ce qui confirme que le basculement final dans l'AutreMonde n'a rien d'incohérent ou de gratuit, comme on l'a souvent suggéré. Ainsi, dès les premiers vers, Rainouart est vu par les païens comme un être enchantés (v. 113) et faeez (v. 120) dont les origines même sont inquiétantes et menaçantes. Aux vers 234 et 270, le texte nous montre le héros tenant à la main un bâton de pommier (arbre aux significations multiples mais convergentes, pouvant symboliser le renouvellement, l'immortalité et un savoir secret réservé aux initiés). Or, cet attribut sera aussi celui de Guiborc (la sœur de Rainouart) au vers 2751 qui se servira justement de cet objet pour agresser violemment Déramé (son père) lorsque celui-ci était sur le point de vaincre son mari (Guillaume). Nous aurons l'occasion de retrouver d'autre indices (liés, notamment, à une certaine conception de la féminité et du temps - motif de l'arbre) au long de ces réflexions.

${ }^{35}$ Ce déplacement a été clairement remarqué et commenté par H. BLOCH (Étymologie et généalogie, p. 217-270) qui en limite toutefois l'impact à l'économie du roman, alors qu'en réalité il me semble affecter également l'économie de la chanson de geste devenant même, à mon avis, l'enjeu essentiel de cette translatio fictionnel de motifs et de séquences appartenant traditionnellement au monde romanesque. Le passage du symbole au signe traduit essentiellement l'éclatement d'un rapport jadis stable et cohérent entre les signes et un signifié transcendant, bien que toujours disponible et récupérable selon l'utopie
} 
exemplairement démontré Sara Kay ${ }^{36}$ à la suite des thèses de Francis Jameson ${ }^{37}$ il est temps d'abandonner ce lieu-commun de la critique qui réduit l'intégration de ces motifs à une simple attitude mimétique de la chanson de geste «contaminé» ou «souillée»par son contact avec ce «vain et plaisant» (et donc éminemment subversif) conte de Bretagne, d'après la célèbre définition de Jean Bodel dans son Prologue à la Chanson des Saisnes ${ }^{38}$. Chansons de geste et romans, formes contemporaines de représentation poétique qui puisent, par conséquent, dans un fond culturel et littéraire commun (qui engendre schémas structuraux et matrices narratives toujours disponibles) ${ }^{39}$, sont plutôt deux figures jumelles qui maintiennent entre elles un intense et puissant rapport intertextuel ou dialogique tissé de rivalités secrètes, d'antagonismes manifestes ou latents, de clins d'œil complices. Ce sont, en quelque sorte, des «fictions politiques»

médiévale (voir la démarche étymologique d'un Isidore de Séville, par exemple) qui désormais devient de plus en plus distant, opaque et intangible. Comme l'explique F. E. SINCLAIR à partir des thèses de J. KRISTEVA, «The symbol thus appears stable and entire, despite the ambiguity perceptible in this referring back to an unknowable transcendence. The monologic quality of the symbol and the gulf which lies between it and that which it symbolises does, however, lend it a self-limiting, self-fulfilling quality; the positive aspects of the symbol are countered by its inherently static, repressive nature. Kristeva sees the thirteenth century as the critical point that marks the beginning of the transition from symbol to sign [...]. The unity and coherence of the relation between the symbol and the transcendence which it evoked began to replaced by 'the strained ambivalence of the sin's connection' with it signified, a distancing which [...] gave rise to the increasingly material nature of the signifying unit (the sign), and to fragmentation and heterogeneity (Milk and Blood, p. 211).

${ }^{36}$ The Chanson de Geste in the Age of Romance. Political Fictions. Oxford: Clarendon Press, 1995.

${ }^{37}$ The Political Unconscious: Narrative as a Socially Symbolic Act. New York, Ithaca: Cornell University Press, 1981.

${ }^{38}$ Même des critiques autrement avisés comme M.-L. Ollier, pour ne citer qu'un exemple, semblent avoir parfois du mal à échapper à ce lieu-commun particulièrement tenace de la mémoire littéraire: «Mais quel qu'ait été le succès du genre, on aperçoit aussi les limites qui lui sont inhérentes; la chanson de geste, comme système de représentation, ne peut pas être protéiforme, comme le sera le discours romanesque; sa primarité s'entend aussi en termes d'unicité; cette forme de discours est historiquement condamnée [...]. Son rôle prend fin dès que, par elle et à travers elle, une communauté s'est reconnue comme telle; dès que chacun de ses membres s'y définit par son appartenance» (OLLIER, M.-L. - La forme du sens. Textes narratifs des XII ${ }^{\mathrm{e}}$ et $\mathrm{XIII}^{\mathrm{e}}$ siècles. Études littéraires et linguistiques. Orléans : Ed. Paradigme, 2000, p. 35). En ce sens, Dans cette perspective, nous sommes à peine surpris par les commentaires de Gerald A. Bertin dans son introduction à l'édition du Moniage Rainouart, lorsqu'il compare ce récit à celui qui va justement faire l'objet de nos remarques, La Bataille Loquifer: «Par contraste avec la Moniage Rainouart, où tout est soigneusement motivé et où la matière épique est préservée malgré les incidents héroï-comiques, La Bataille Loquifer, qui est moitié moins longue (3890), est caractérisée par une suite médiocre d'évènements mal liés où le merveilleux et les coïncidences réduisent l'épopée au niveau du roman d'aventure banal»(BERTIN, Gerald A. - Le Moniage Rainouart I. Paris : Éd. A. \& J. Picard \& $\mathrm{C}^{\mathrm{ie}}, 1973$, p. LV-LVI).

${ }^{39}$ Voir ROUSSEL, Cl. -«Le mélange des genres dans les chansons de geste tardives», in ALVAR, C. et PAREDES, J. - Les Chansons de geste. Actes du XVI ${ }^{\mathrm{e}}$ Congrès Internationale de la Société Rencesvals pour l'Étude des Épopées Romanes (Granada, 21-25 juillet 2003). Granada: Université de Granada, 2005, p. 73. 
placées sous le signe de Janus, offrant ainsi une vision/lecture différenciée du monde et de l'écriture ${ }^{40}$. La confluence qui dès lors se produit engendre une véritable écriture réflexive (ou de second degré) où la geste, en se rêvant comme un roman (comme il arrive dans La Bataille Loquifer), interroge à la fois le pouvoir et les limites de ces deux systèmes poétiques de représentation et la vision du monde qui sous-tend chacun d'entre eux. Or, le motif du baume magique condense justement l'attitude ambigüe de la chanson à l'égard du sang et le rapport privilégié que se fluide semble entretenir avec une conception singulière de l'écriture poétique. Alternativement mis au service du héros chrétien et du héros sarrasin, ce baume que Rainouart finira par usurper à Loquifer et dont les multiples occurrences (au moins dix entres les vers 1588 et 2308) sont elles-mêmes surprenantes est en effet extrêmement paradoxale, car si, d'une part, il permet la constante restauration de l'intégrité (l'imperméabilité) du corps ${ }^{41}$, étanchant l'hémorragie de l'écriture et du sens qui caractérise et menace généralement le registre épique, il condamne néanmoins, une fois de plus, la chanson de geste à l'infinie répétition du même, à l'inachevable. S'emparer de cet onguent confère, comme on peut s'en douter, à son détenteur un pouvoir extraordinaire: pouvoir divin de donner la vie et la mort, de gommer le spectre de la souillure et du temps ${ }^{42}$, de choisir - choix ô combien délicat et impossible - entre un père symbolique marqué cependant par la défaillance (image d'un Guillaume fragile en cette fin de cycle, constamment pessimiste sur le destin de la Chrétienté et qui sera sauvé in extremis de la mort grâce à l'intervention de sa femme Guiborc ${ }^{43}$ ) et une paternité biologique rejetée (Déramé), c'est-à-dire de décider du devenir de la geste (famille et discours poétique), et, finalement, pouvoir strictement poétique ou auctorial d'interrompre ou de poursuivre

\footnotetext{
${ }^{40}$ En s'aventurant dans l'univers arthurien, la chanson de geste peut ainsi surgir comme une sorte d'inconscient poétique (et idéologique ou politique) du roman, traduisant (au sens médiéval de la translatio, d'un déplacement discursif et métaphorique des signifiants fictionnels), questionnant ou rendant manifeste ce que celui-ci a voulu réprimer au niveau du dit, Comme le suggère $\mathrm{S}$. Kay (The Chanson de geste in the age of the romance, p. 6), le roman, par exemple, semble mettre en scène «a politics of evasion which sanitize or disguise the rifts in the social and symbolic order which chansons de geste exhibit.»

${ }^{41}$ Face au spectre de la fragmentation et du morcellement du corps (physique et poétique) qui menace la chanson de geste, la présence du baume magique alimente le rêve d'une unité/intégrité infiniment retrouvée: «n'i parut plaie an ses jambes ne trous» (v. 2310).

${ }^{42}$ L'épisode biblique (rapporté par Matthieu et par certains apocryphes) racontant la guérison, par Jésus, de la femme (Véronique, d'après L'Évangile de Nicodème) soufrant d'hémorroïsse est, dans cette perspective, très révélateur. Voir, à ce sujet, les commentaires de ROUX, J.-P. - Le Sang..., p. 88-90.

${ }^{43}$ Sans parlé de l'autre figure de la Loi (absente de ce texte) qui incarne la faille durant tout le cycle de Guillaume d'Orange, à savoir, le roi Louis le Pieux (voir SINCLAIR, F. E. - Milk and Blood, p. 208-210).
} 
indéfiniment le récit. Refuser l'épanchement de sang, nier le morcellement du corps, éviter la mort, c'est donc suspendre le chant épique en le condamnant, à nouveau, virtuellement à l'aporie.

Que le récit cherche à différer (dans l'attente d'une issue quelconque) la résolution du combat entre ces doubles que sont Rainouart et Loquifer ${ }^{44}$ et, dans l'autre versant (celui des pères), Guillaume et Déramé, cela est parfaitement compréhensible, l'enjeu des duels (surtout celui du premier) étant aussi bien d'ordre eschatologique qu'idéologique, symbolique et textuel. Eschatologique et idéologique, dans la mesure où la mort de Rainouart et de Guillaume impliquerait la désintégration du monde féodal et de logos chrétien sur lequel il se fonde ${ }^{45}$. Symbolique et textuel, dans la mesure où ce conflit laisse entrevoir la tension entre deux modèles discursifs et culturels qui s'appuient habituellement sur des strates et des logiques distinctes de l'imaginaire. Remarquons, en effet, que les trois combats décisifs de Rainouart qui structurent le récit sont tous liés, d'une façon ou d'une autre, à une généalogie de l'Autre-Monde. Le sang qui coule dans les vaines d'Ysabras, le cruel guerrier qui conduit les opérations contre Rainouart au début du récit, est de nature féérique: ayant subit un enchantement à sa naissance, ce personnage, décrit comme une être difforme («moult l'avoient diversement figuré», v. 198), a en effet été condamné à vivre sous l'aspect d'un monstrueux noitons durant trente ans (v. 193-204). Remarquons d'ailleurs que c'est également un luiton, nommé Grigalet, qui a violé Bruneholt sur l'île d'Orion et engendré Chapalu (laisse 82), cette chimère que Rainouart devra affronter à Avalon et qui ne recouvrera sa forme humaine

\footnotetext{
${ }^{44}$ Les deux personnages ont en effets plus de points en communs que de différences: tous deux sont présentés comme des géants aux attributs telluriques ou chtoniens; tous deux refusent la plupart du temps de combattre avec l'emblème par excellence du guerrier, l'épée, préférant la massue (Rainouart) et la loque (Loquifer), une arme redoutable dont le héros s'emparera à la fin du combat prenant alors clairement la place de son double; tous deux ont pour habitude de s'asseoir «sous l'olivier ramé» (tout comme le fera d'ailleurs Guiborc lors du duel entre Guillaume et Déramé), cet axis mundi - qui symbolise la paix ou l'alliance - devenant naturellement un attribut de tous ces personnages de frontière que sont Rainouart (le géant païen converti et devenu champion de la chrétienté), Guiborc (la belle sarrasine usurpée par Guillaume à Thibaud) et Loquifer (dont l'espace d'origine est clairement présenté sous les traits de l'Autre-Monde).

${ }^{45}$ Le texte insiste à plusieurs reprises sur cette menace imminente: sans Rainouart, la geste de Guillaume (et par conséquent la Chrétienté carolingienne) est perdu (voir, par exemple, le planctus de Guillaume aux vers 2075-93.
} 
qu'après avoir bu le sang du talon du héros. Le motif celtisant du viol de la fée ${ }^{46}$ rejoint ici celui, précédemment repéré, de la violence épique exercée sur le corps féminin. À la laisse XXVIII, nous apprenons encore qu'une partie de l'armure du géant Loquifer a été confectionnée dans l'Autre-Monde (v. 1552 sq.) et que ses adjuvant (aux noms suffisamment éloquents de Pilate et Belzébuth: v. 2379) viennent d'outre les Bornes Arthur (v. 2381), espace qui marque probablement la frontière avec l'Autre-Monde d'Avalon où, selon la tradition poétique reprise par ce texte, le roi mourant s'est retiré auprès de sa sœur Morgue. Le combat entre les deux champions se situe d'ailleurs sur une île (espace étranger à la tradition épique que l'on retrouvera néanmoins dans le Moniage Rainouart) au large de Porpaillart et commence un mercredi (le milieu de la semaine) à midi (l'heure, extrêmement délicate, de l'ouverture entre les deux mondes, l'heure du passage par excellence ${ }^{47}$ ). Sous le voile de la métaphore fictionnelle, tous ces combats relèvent donc, en fait, d'un antagonisme idéologique et imaginaire entre le monde épique et l'univers du merveilleux breton. Comme le montre exemplairement le cas du baume, s'emparer de la merveille profane (le miracle chrétien - transformé en une espèce de topique figé - étant ici presque réduit à néant), dont le roman a très vite compris les ressources inépuisables, devient, dans La Bataille Loquifer, une stratégie habilement menée pour éviter la fragmentation du corps épique dont la matière et la forme tendent à s'épuiser.

Ce processus de déplacement et de conversion est particulièrement mis en relief par certains éléments narratifs, liés directement ou indirectement au sang, qui scandent la partie épique du récit. Remarquons tout d'abord que Rainouart ne s'empare pas seulement de l'onguent magique de Loquifer, mais également des trois épées du géant sarrasin. Or, si le baume avait pour fonction d'étancher le sang et de suspendre

46 Voir, à ce sujet, VINCENSINI, J.-J. - «Viol de la fée, violence du féerique. Remarques sur la vocation anthropologique de la littérature médiévale». Senefiance, 36 (1994), p. 545-559. N'oublions pas, d'autre part que Maillefer, le fils d'Aélis et de Rainouart naît, lui aussi, sous le signe de la démesure physique et que sur lui plane aussi, à en croire les insultes des païens et les étranges coïncidences lexicales que nous avons observées, le spectre de la bâtardise. Vaincre la chimère conçue dans l'Autre-Monde, c'est donc également exorciser la menace d'une généalogie chrétienne placée sous le signe de la monstruosité.

${ }^{47}$ Voir STANESCO, M. - «Du démon de midi à l'Éros mélancolique: topologie du féerique dans le lai narratif breton». Poétique, 106 (1996), p. 131-159. 
indéfiniment l'hémorragie et le récit épique lui-même, le second motif, lié au schème de la diérèse, de la coupure, doit, au contraire, faire couler le sang afin de restaurer la dynamique du temps et le cycle de la vie et de la mort. Mais encore faut-il noter la nature singulière de ces trois épées ${ }^{48}$. Il s'agit de Recuite «qui Alixandre fu», de Douloureuse «qui fut Roi Chapalu» et de Hideuse «qui fu faite a Val Bru/ c'est une terre ou li home sont nu» (v. 2394-98). Cette singulière trilogie représente, nous le devinons, une véritable trinité poétique, chaque épée s'érigeant en emblème d'une matière narrative particulière (la matière antique avec Alexandre, celle de Bretagne avec Chapalu et la matière de la chanson de geste traditionnelle avec Hideuse façonnée dans l'espace païen de Val Brun $^{49}$ ). Faut-il s'étonner qu'à ce moment du récit la matière arthurienne incarnée par Douloureuse émerge comme le miroir inversé de la matière carolingienne représentée par Joyeuse? Est-ce également par simple coïncidence si le héros coupe la tête de Loquifer à l'aide de Recuite dont le nom fait miroiter l'image du feu régénérateur (l'élément archétypal de Rainouart) associée à celle du forgeron, support métaphorique bien connu de l'activité scripturaire qui se présente ici comme refonte de matériaux de diverses natures et origines, i.e., comme acte de réécriture par excellence (la translatio dont résulte le roman antique étant justement l'emblème achevé de cette opération)? Ou alors, La Bataille Loquifer insinuerait-elle plus simplement que seule une matière étrangère et au roman et à la geste est susceptible de faire renaître la tradition épique? Ce discours méta-poétique subliminaire est d'autant plus important que la séquence de l'appropriation des trois épées (laisses 45-46) se situe peu avant le court prologue intercalé qui attribue l'inventio de la chanson à un dénommé Jendeus de Brie (laisse 49), épisode qui est lui-même suivi (dans la même laisse et la laisse suivante) de l'éloge tissé à Joyeuse comme modèle (ou pseudo-modèle à l'instar de cette source fictive) de l'intégrité de la mémoire épique carolingienne. Si la mort de Loquifer témoigne de la nature omnivore d'une écriture épique qui, au XIII ${ }^{\mathrm{e}}$ siècle, tend à s'alimenter d'une diversité croissante de sources et de ressources narratives, celle de

\footnotetext{
${ }^{48}$ Sur cette question dans le contexte de la confluence inter-générique qui caractérise ce poème, voir nos réflexions dans «Rainouart au pays des fées. Interchangeabilité des personnages et dialogisme dans "La Bataille Loquifer"», in CONNNOCHIE-BOURGNE, Ch. - Façonner son personnage au Moyen Âge. Senefiance, 53. Aix-en-Provence : PUP, 2007, p. 99-122.

${ }^{49}$ Outre le fait que la laideur soit un attribut caractéristique de la description des païens dans l'épopée traditionnelle, remarquons que, dans la Chanson de Roland par exemple, de nombreux Sarrasins exhibent un nom construit sur le morphème val- (la vallée symbolisant souvent les profondeurs abyssales de l'enfer). C'est le cas de Justin de Valferee à la laisse 107 ou celui de Valdabrun à la laisse 117 (éd. critique SHORT, I. Paris : Librairie Générale Française, Coll. Lettres Gothiques, 1990).
} 
Déramé côtoie le mythe de fondation bâti sur les vestiges d'anciennes croyances concernant la dangereuse rencontre entre deux fluides apparemment contraires: le sang impure et l'eau purificatrice ${ }^{50}$. En effet, après avoir été érigée en totem et suspendue à un poteau à l'entrée de la salle du palais de Guillaume à Orange, la tête de Déramé dont la force diabolique continue à troubler l'ordre cosmique (des tempêtes incessantes fustigent la ville) - est lancée dans la mer afin de conjurer l'angoisse qui émane encore de la présence du sang/âme de ce personnage qui fut à l'origine d'une prolifératrice généalogie du mal. Or, à en croire la (pseudo-)légende rapportée par le texte, nul navire n'ose désormais passer à l'endroit où elle fut jetée - qui reçut le nom de Salt Malatois de peur de naufrager (v. 3525-39) ${ }^{51}$.

Plus loin dans le poème, sur un récit qui se déroule en second plan, l'alliance du sang et de l'eau s'enchérit par l'apport d'un autre fluide aussi ambigu que le premier. Le texte nous raconte en effet que le nain Picolet lou ligier (v. 965), le messager au service de Loquifer $^{52}$ et maître ès-art de l'enchantement, enlève Maillefer pour l'emporter à

\footnotetext{
50 Comme il arrive fréquemment dans le fonctionnement du symbolique, l'antithèse peut devenir complémentarité par l'union des contraires. Ainsi, dans certains contextes rituels, le sang est aussi purificateur que l'eau (tous deux sont principes de vie). Versé sur la terre qu'il féconde, il suscite des pluies abondantes, à conditions qu'il s'agisse d'un sang vivant, chaud et clair (voir ROUX, J.-P. - Le sang..., p. 83-88). Cependant, lorsqu'il s'agit d'un sang considéré impur (celui des menstrues ou de la parturition, notamment), son contact avec l'eau est susceptible de provoquer de terribles tempêtes (sur la résurgence de ce motif dans la littérature médiévale, je renvoie aux commentaires de P. MCCRACKEN The Curse of Eve, p. 53-58.

${ }^{51}$ Remarquons que cet imaginaire de la régénération est également présent au long du récit à travers quelques indices discrets (mais révélateurs) qui assument de nets contours mythiques. Représentant une nouvelle lignée de héros, Rainouart est comparé, au vers 2340, à un «faucons de mue». Plus tard, la comparaison à valeur métaphorique prendra corps littérale dans la fiction lorsque les fées, emportant le héros à Avalon, transforment sa massue en un faucon (v. 3655). Notons encore que Rainouart est souvent présenté assis sous un olivier ramé et que le bienfaisant nain Picolet qui sauvera Maillefer est deux comparé à un «cerf ramé» (v. 2121 et 2561), symbole celtique bien connu de la rénovation associée au dieu Cernunos. À la lumière de tous ces indices qui soulignent le schème de la renaissance (de la chanson de geste, du lignage, de la Chrétienté, etc.), ne pourrait-on pas interpréter le nom de Des-ramé comme l'emblème d'un univers épique usé, stérile (comme, de l'autre côté du miroir, l'est également celui de Louis le Pieux) et, par conséquent, condamné à disparaître?

${ }^{52}$ Bien que le texte ne le dise pas explicitement, son nom révèle qu'il s'agit bien d'un avatar du nain («Petit estoit», v. 3314), miroir négatif et complémentaire du géant, dont la présence scande les récits arthuriens. Les trois yeux (v. 966-967) qu'il exhibe en font une figure de la totalité qui joint les différents axes séparés de l'espace et du temps (passé, présent et futur), tout en évoquant, peut-être, les trois matières poétiques métaphorisées par les trois épées de son maître. Quant aux brides que le poème nous fournit sur la généalogie de ce personnage, elles sont également révélatrices de son appartenance à un domaine culturel et poétique autre que celui de la chanson de geste traditionnelle. Nous savons, en effet, qu'il est frère d'Aubéron «qui de Monnuble tenoit la regïon» (v. 3315). Ce domaine, dont Picolet se
} 
Loquiferne (laisse 48). Désirant venger la mort de Loquifer qu'elle a alimenté de son propre sein, une monstrueuse et perverse nourrice est sur le point de le condamner au bûcher avec l'accord tacite de Thibaud qui refuse de souiller ses mains (et son image) par cet abominable crime. Picolet s'apitoie du sort de cet enfant qui présente tous les signes du héros exceptionnel et prédestiné ${ }^{53}$. C'est alors qu'il parvient, par l'effet d'un philtre magique, à l'enlever une nouvelle fois pour le transporter dans son domaine d'origine, Monnuble, ce non-lieu introuvable de la géographie arthurienne planté en plein territoire sarrasin et épique. La haine de la nourrice à cependant une autre cause, bien plus importante en ce qui concerne le sujet de cette réflexion. En effet, depuis qu'il est à sa garde, Maillefer refuse de s'allaiter, préférant jeûner et se nourrir d'une extraordinaire quantité d'eau que de toucher au sein de la païenne:

\author{
«Par Mahomet, que je l'ai acorneté! \\ A ma memelle n'a tochié n'adesé, \\ ne la presist por .M. mars d'or pesé; \\ par felonie a mainte fois juné, \\ mais d'eve boit .I. sester mesuré; \\ mal soit del baing que li aie tenpré» (v. 4033-38).
}

Contrairement à ce qu'affirme la nourrice, ce n'est pas seulement «par félonie» que Maillefer se comporte de cette façon. Suivant une tradition médicale et théologique solidement attestée et enracinée au Moyen Âge ${ }^{54}$, nous savons que le lait n'est autre que du sang menstruel blanchi après avoir subi une forte coction durant la grossesse, la seule

présente comme le légitime héritier, se situe apparemment dans l'espace balisé du monde païen tout en échappant à sa géographie imaginaire, ce qui explique pourquoi Thibaut échoue à retrouver Maillefer bien qu'il le fasse rechercher partout (v. 4137-39). Il est, d'autre part, intéressant d'observer que la digression narrative qui raconte ce nouveau rapt de Maillefer emporté maintenant vers l'Autre-Monde de la féerie et du roman arthurien, se place justement au cœur de la séquence d'Avalon (laisses 89-91) et non pas dans la partie épique du poème. Picolet le nain apparaît ainsi comme une figure médiatrice par excellence qui opère un premier déplacement géographique et métaphorique (une véritable translatio poétique) de la chanson de geste vers l'espace romanesque.

${ }^{53}$ Le rire sardonique de l'enfant (v. 4021) n'est pas sans rappeler celui de Merlin et reprend le motif très répandu au Moyen Âge du puer senex.

54 Voir MCCRACKEN, P. - The Curse of Eve, p. 4-6; THOMASSET; Cl. - «De la Nature féminine», p. 78-84; SINCLAIR, F. - Milk and Blood, p. 17-27; 32-42. 
période où la femme, froide et humide par nature, possède plus de chaleur naturelle. Le lait est donc toujours le résultat d'une purification du sang dont il conserve néanmoins certaines caractéristiques, l'une des plus importantes étant précisément les traits (physiques et moraux) de la mère/nourrice qui peuvent ainsi se transmettre héréditairement à l'enfant. Or, outre le fait qu'il s'agisse d'une païenne, c'est surtout de la nature monstrueuse (n'oublions pas que ce fut elle qui a alimenté Loquifer!) et infanticide de cette nourrice - modèle de le bestialité aussi bien qu'avatar de la sorcière du folklore - que Maillefer cherche sagement à préserver son corps et son âme:

Es la norrice, ses cors soit vergondés que plus est noire que aremens triblés; grant ot la gole, demi piet mesuré; de ses mamelles vos dirai verité, en .I. lit ot .VI. paien engendré; li .III. sont mort, et li .III. sont remés. Chascons estoit de Maillefer ans né; sous qui sont mort ot a ses poinz tüés, et au .III. autres chascon les iolz crevés; de ses joiaus lor avoit ja mostré (v. 4063-72).

Plus énigmatique, le choix de l'eau semble néanmoins se justifier par une double motivation: face à l'image du sang/lait contaminé et potentiellement corrupteur, l'eau est non seulement un symbole quasi universel de la pureté présent dans la plupart des rites de purification, comme elle permet à l'enfant de se rattacher aux origines paternelles dont il ignore cependant tout. En effet, l'eau renvoyant, d'une part, au rituel baptismal auquel se sont soumis Rainouart et Guiborc (tante de Maillefer), elle préfigure ou confirme l'appartenance de l'enfant à l'ordre chrétien. Mais ce fluide évoque ou convoque, d'autre part, grâce au pouvoir de l'intertexte, le passé du père qui, avant de devenir le guerrier au service la geste carolingienne, fut acheté par Louis le Pieux à des marchands et enfermé durant sept dans les cuisines du palais, l'une de ses 
fonctions consistant justement à porter d'énormes quantité d'eau suspendus de chaque côté de son inséparable tinel ${ }^{55}$.

\section{Achille chez les vampires: les avatars du sang épique}

Située au carrefour de la tradition épique et romanesque, du mythe et du folklore, La Bataille Loquifer met en scène un imaginaire complexe, diversifié et parfois paradoxal du sang sur lequel se projette à la fois le devenir du lignage (menacée de dispersion) et les enjeux de l'écriture épique dont il semble épouser les mouvements et les formes symboliques, une convergence qui culmine évidemment avec l'épisode d'Avalon et l'étrange personnage de Chapalu. Le schème de la régénération est désormais pris en charge par la narration même qui, renaissant de ses cendres, annonce une «chanson anluminee/ [qui] ans de jugler ne fut mellor chantee» (v. 3569-70) qui, à en croire la tradition manuscrite de cette séquence, fut parfois considérée comme une présence troublante et menaçante à l'égard de l'identité textuelle et idéologique du poème ou de la compilation qui l'a recueille ${ }^{56}$. Aussi, l'entrée au Royaume de Féerie représente-t-elle un moment particulièrement délicat pour le récit qui se voit alors dans l'obligation de déployer l'habituelle rhétorique de la vérité de l'estoire ancrée dans une pseudo-source écrite («Verités est, ce tesmoigne l'escris», v. 3602) ${ }^{57}$. Quels sont les enjeux du ravissement de cette chanson de geste qui soudain se rêve (ou serait-ce plutôt un cauchemar?) comme un conte de Bretagne. Remarquons que si ce voyage de Rainouart, apparemment gratuit et sans conséquences, a pour but de régénérer un discours qui, tout

\footnotetext{
55 «.IIII. muis d'eve li ai veü porter/ A un tinel et a son col lever», apprend-on de la boucle du roi Louis dans les Aliscans (v. 3583-84).

${ }^{56}$ En effet, l'instabilité dérangeante de l'épisode d'Avalon est visible à travers les différentes versions du texte: certains manuscrits (Arsenal, F, E et peut-être C) effacent cette séquence, alors que d'autres la dissocient du reste du texte (B2) ou l'associent directement aux premiers vers du Moniage Rainouart (B1), les versions qui donnent cet épisode présentant néanmoins entre elles une homogénéité remarquable. Soigneusement préparé tout au long du récit par une constellation extrêmement cohérente du point de vue narratif et symbolique, cette séquence, comme nous avons cherché à le démontrer, est toutefois loin d'être un simple collage maladroitement greffé (par un quelconque remanieur) sur un récit premier (au plus, si greffe il y eut, elle a pris le soin de tenir compte - ou d'introduire - certains thèmes et motifs parsemés tout au long du poème). Sur la place qu'occupe La Bataille Loquifer dans le cycle de Guillaume d'Orange et, plus particulièrement, sur la mouvance matérielle qui affecte l'épisode d'Avalon dans la tradition manuscrite, je renvoie à l'excellente étude de Nelly ANDRIEUX - «Arthur et Charlemagne réunis en Avalon: la Bataille Loquifer ou l'accomplissement d'une parole», in Actes du IX Congrès International de la Société Rencesvals. Modena : Mucchi, vol. 2, 1984, p. 425-434.

${ }^{57}$ Par opposition à l'image du «livre faussé» par les sarrasins qui apparaît à la fin de la laisse VI.
} 
comme dans la première partie de La Chanson de Guillaume ou celle d'Aliscans, semble souffrir d'épuisement (à l'image du héros s'endormant sous cette figuration de l'axis mundi qu'est l'Arbre qui Fant - v. $3635^{58}$ ), il prétend également confronter la matière arthurienne avec ses propres limites ou limitations. Il n'est donc pas étonnant que la séquence d'Avalon s'ouvre sur une métamorphose qui relève clairement de la conversion poétique. Les attributs qui façonnent habituellement Rainouart comme personnage épique sont transformés par les fées en objets qui renvoient à l'univers courtois, le tinel devenant un faucon, le haubert un jongleur gascon ${ }^{59}$ et le heaume cinquante bretons qui entonnent avec douceur le lai de Goron, l'épée devenant un garçon (laisse 75). Remarquons que la métamorphose ne se limite pas à produire (ou à reproduire) une structure de type romanesque, mais oblige la matière arthurienne à remonter à ses sources orales fondatrices pour s'y régénérer (comme l'a fait Marie de France dans les Lais). Ce dépassement de la tradition scripturaire transmise par Geoffroy de Monmouth, Wace ou Chrétien de Troyes est bien visible lorsque l'une des fées présente Rainouart à Arthur comme étant le meilleur champion «qui onques fust en fable n'en chanson» (v. 3669). Ce renvoi à une source lointaine et dont l'auctoritas est toujours suspecte - une source d'où émerge également le personnage de Chapalu -, est donc extrêmement ambigu, à l'instar des nombreuses notes dissonantes qui caractérisent le voyage à Avalon, à commencer par la minutieuse description de ce séjour qui, dans les romans ou dans les lais, se place toujours sous le signe de l'inter-dit, du silence ou de l'ellipse narrative. Que penser également du rire sardonique et merlinien d'Arthur (qui semble gouverner Avalon - «par lou conment Artu», v. 3741 - aux côtés de sa sœur Morgue - conférant à l'ultime combat rituel entre Rainouart et Chapalu l'allure d'un divertissement, d'un immense gab (laisse 81), aux contours toutefois éminemment initiatiques.

\footnotetext{
${ }^{58}$ Symbole qui s'inscrit dans la lignée de tous ces arbres «ramés» qui parsèment le récit et sous lesquels les personnages se reposent à tour de rôle (Loquifer, Rainouart, Giborc, Ygerne).

${ }^{59}$ Tout lecteur/auditeur attentif des prologues épiques et romanesques des $\mathrm{XII}^{\mathrm{e}}$ et $\mathrm{XIII}^{\mathrm{e}}$ siècles sait néanmoins qu'il faut toujours se méfier des histoires rapportées oralement par ces jongleurs (surtout lorsqu'ils sont gascons?) qui n'ont cesse de déformer et de corrompre la vérité du conte, de briser l'intégrité de la parole poétique.
} 
Le séjour à Avalon se structure en effet entièrement autour d'un étrange rite de sang. Comme nous l'apprenons dans la laisse 82, Chapalu, personnage engendré par la fée Bruneholt (mère de Morgue) à la suite de la transgression d'un double interdit par le luiton Gringalet (la fée est surprise dans son bain - nous reconnaissons-là un schème typiquement mélusinien ${ }^{60}$ - et violée «an traïsson»), hérite du péché de son père et est condamné par la fée à prendre la forme d'une chimère (yeux rouges, tête de chat grosse et velue, pieds de dragon ou de léopard, ongles de griffon, corps de cheval et queue de lion: laisses 79-80 et 82), jusqu'à ce «qu'il eüst bu del sanc del talon/ de Renoart, lou mellor chanpïon» (v. 3815-16). Trois questions se posent. Tout d'abord, d'où provient et que signifie ce singulier personnage? Ensuite, quelle valeur et quelle fonction le sang assume-t-il au sein de ce rituel vampirique? Finalement pourquoi le fluide doit-il couler à partir d'une blessure située au talon de Rainouart? Chapalu est un être aussi paradoxal que son apparence: appartenant indéniablement à l'univers breton, il est néanmoins étranger à la tradition manuscrite canonique telle qu'elle est transmisse par Geoffroy de Monmouth, Wace ou Chrétien. La forme française Chapalu résulte probablement d'une déformation de Cath Palug, le monstre marin à forme de chat (scénario qui se reflète encore dans La Bataille Loquifer où ce sera en mer que Chapalu essayera de tuer Rainouart) qui apparaît pour la première fois dans un poème arthurien du VIII ${ }^{\mathrm{e}}$ ou $\mathrm{IX}^{\mathrm{e}}$ siècle intitulé $\mathrm{Pa}$ gur yv y porthaur basé lui-même sur une tradition orale antérieure. Il devait cependant être parfaitement connu à l'époque où le texte fut composé, vu que la première mention à cette figure dans La Bataille surgit discrètement dans la bouche de Rainouart à travers une expression à valeur proverbiale («Dahait ait ons qui croit en Chapalu», v. 1629), c'est-à-dire, déjà cristallisée dans la langue. D'autre part, Chapalu émerge également dans un texte normand de la fin du XII ${ }^{\mathrm{e}}$ siècle attribué à André de Coutances, Le Romanz des Franceis, où il perd cependant une partie de sa force mythique et mythologique étant mis au service d'un discours satirique dirigé contre les Français par un sujet normand des Plantagenêt (d'où la parodie burlesque de la conquête de la France par le roi Arthur qui inaugure le récit). Outre les toponymes auxquels la légende a donné naissance (le $\mathrm{Col}$ du Chat, le Mont du Chat), d'autres textes en langue

\footnotetext{
${ }^{60}$ Sur l'isomorphisme possible entre le bain rituel (marqué par l'interdit) et les tabous concernant le sang menstruel, voir notamment l'étude d'ISNART, C. - «La fée, la grotte et le tisserand. Etude ethnologique autour de la Monographie communale d'Utelle [circa 1910]». Pays Vésuvien, 1 (2000), p. 147-157, ainsi que les commentaires de MCCRACKEN, P. - The Curse of Eve, p. 79-91).
} 
vernaculaire y font référence, en liant néanmoins toujours cette figure au domaine breton. C'est le cas «Dou miracle qui avint aus Bretons de Chartres» ${ }^{61}$ ou d'un passage de Galeran de Bretagne (XIIIe siècle) qui fait référence au roi Arthur comme étant celui «Que le chat occist par enchaus» (v. 5071) ${ }^{62}$, suggérant ainsi l'existence d'une tradition/légende différente de la mort d'Arthur (que notre texte ferait miroiter à travers Hideuse, l'épée qui a appartenu au roi Chapalu: v. 2396) de celle que nous présentent les versions «canoniques» (où Arthur meurt suite à une blessure infligée par Mordret durant la bataille de Salesbiere, étant alors transporté dans l'île d'Avalon pour être guéri par sa sœur Morgue). Cela expliquerait en partie la relation privilégiée qui existe, dans La Bataille Loquifer, entre Arthur et $\mathrm{Chapalu}^{63}$ et le fait que celui-ci soit en quelque sorte à l'origine des enchantements qui règnent sur Avalon qui s'identifie alors clairement avec l'Autre-Monde la mort que Rainouart a pour fonction de ramener à la $v_{i e}{ }^{64}$. Mais, à l'instar d'autres motifs qui parsèment le récit, Chapalu commence par être une image cristallisée dans la langue proverbiale ou une réminiscence fictionnelle lointaine. Il est donc avant tout un signifiant poétique qui soudain s'amplifie (rhétorique de l'amplificatio), s'autonomise et finit par incarner dans la fiction, devenant emblème et/ou métaphore de l'hybridisme qui caractérise aussi bien Rainouart ${ }^{65}$ que cette chanson de geste.

\footnotetext{
${ }^{61}$ Ce miracle raconte comment la Vierge vient en aide aux Bretons en les guidant dans l'obscurité lorsqu'ils se dirigent avec leurs chars à Chartres pour reconstruire une église en son honneur: «Hors ne furent pas li Breton/ De la mesnie Chapalu» - v. 94-95: édition en ligne du ms. Chartres 1027 par KUNSTMANN, P. (consulté le 26 août 2010). Disponible à l'adresse WwW.uottawa.ca/academic/arts/lfa/activites/textes/chartres/chpres.html.

${ }^{62}$ Éd. L. FOULET, L. Paris : Champion, 1975. Sensiblement à la même époque (ce qui n'est peut-être pas le simple fruit du hasard), nous retrouvons également des échos de cette tradition dans la Suite-Vulgate du Merlin (chap. 32-33), en particulier lors du combat civilisateur qui oppose Arthur au chat démoniaque.

${ }^{63} \mathrm{C}$ 'est en effet sous les ordres du roi que le monstre hybride est libéré pour combattre Rainouart: laisse 79; d'après les paroles d'une fée, le souverain semble d'ailleurs être le seul, en dehors de la victime, à connaître la forme humaine de Chapalu.

${ }^{64}$ Nous retrouverons le personnage de Chapalu plus tard, notamment dans les remaniements d'Ogier le Danois (version décasyllabique du XVe siècle) où son origine et son rôle sont présentés dans une version assez semblable à celle que nous trouvons dans la Bataille Loquifer. Voir, à ce sujet, les pertinentes remarques R. TRACHSLER- Disjointures - Conjointures. Etude sur l'interférence des matières narratives dans la littérature française du Moyen Âge. Tübingen-Basel : A. Francke Verlag, Romanica Helvetica, 2000, p. 150-156.

${ }^{65}$ Être de l'oxymore ou de la conjonction (aussi bien idéologique que symbolique) des contraires par excellence, Rainouart est le géant païen qui, bien qu'étranger au monde épique traditionnel, devient champion de la Chrétienté; il est aussi le héros de la troisième fonction dumézilienne qui sort de l'univers souterrain des cuisines pour s'ériger au statut de prince - marié à la fille de Louis le Pieux - et de seigneur féodal).
} 
Deuxième question: pourquoi le retour de Chapalu à la forme humaine (c'est-à-dire, à la Figura moulée par Dieu à son image) implique-t-elle l'ingestion du sang de Rainouart? Commençons par l'aspect le plus évident. Si le sang contient des principes qui ne s'altèrent pas et s'il est le véhicule privilégié des qualités de l'homme, de son âme, de son énergie vitale, il est d'autant plus précieux, efficace et régénérateur qu'il prend sa source dans un corps valeureux (le meilleur guerrier du monde) qui transmettra ainsi toutes ses vertus à Chapalu. Toutefois, l'absorption de ce fluide ne va pas de soi pour la civilisation médiévale. Cette coutume, propre des païens et des barbares et sur laquelle retombe divers interdits bibliques (Lévithique, XVII, 10-14, par exemple) ${ }^{66}$, représente, $\mathrm{du}$ point de vu anthropologique, une régression culturelle vers l'informe et la bestialité ${ }^{67}$. Signalée avec dégoût par Isidore de Séville dans son Historia Gothorum, elle sera sévèrement condamnée par les pénitentiels comme l'un des crimes les plus abominables qu'il soit ${ }^{68}$. Par opposition au sang pur et rédempteur du Christ déversé sur la Croix et consommé à travers le sacrement commémoratif de l'eucharistie, l'ingestion du sang humain est également, au Moyen Âge, et suivant une tradition bien connu depuis l'Antiquité, œuvre des démons qui cherchent à perdre les hommes sous forme de striges ou de lamiae (que celles-ci assument les contours des sylvains, des pans, des incubes ou même de ces créatures fantasmagoriques que l'on dénomme fées), c'est-àdire, de «visions nocturnes qui, du fait de l'épaississement des humeurs, troublent les âmes des dormeurs et les écrasent de leur poids». Cette définition proposée par Gervais de Tilbury dans son Livre des Merveilles (Otia Imperalia, III, 86) ${ }^{69}$, composé au tournant du XII ${ }^{\mathrm{e}}$ et du XIII ${ }^{\mathrm{e}}$ siècle (donc contemporain de La Bataille Loquifer), est particulièrement intéressante dans la mesure où il compte les fées parmi ces créatures fantasmagoriques (Rainouart est emporté à Avalon par quatre fées), où il indique clairement que celles-ci agissent durant le sommeil (c'est encore le cas de notre héros)

\footnotetext{
${ }^{66}$ Pour un relevé plus complet des sources judéo-chrétiennes et antiques, voir l'ouvrage de ROUX, J.-P. Le Sang: mythes, symboles et réalités.

${ }^{67}$ Comme le souligne J. VOISENET («Le tabou du sang dans les pénitentiels du haut Moyen Âge», in Le Sang au Moyen Âge, p. 117), «Le sang n'est plus seulement le vecteur de vie, la chair crue et sanguinolente symbolise la bestialité et le paganisme. Sa condamnation met nettement en valeur l'opposition entre le cuit et le cru, la culture et la nature, la civilisation et la sauvagerie. Absorber le sang, sous n'importe quelle forme, c'est franchir cette limite incertaine entre l'humain et le bestial, c'est renoncer à l'élévation du christianisme pour retomber dans le paganisme.»

${ }^{68}$ Voir l'intéressant article de J. VOISENET, «Le tabou du sang dans les pénitentiels...», p. 111-125.

${ }^{69}$ Texte traduit et commenté par DUCHESNE, A. Paris : Les Belles Lettres, 2004.
} 
et, finalement, dans la mesure où il affirme que l'une des caractéristiques essentielles de ces démons consiste dans l'ingestion de sang humain:

On les voit aussi manger, allumer les lumières, disjoindre les os des hommes pour les remettre parfois dans un autre ordre, boire le sang humain et déplacer les petits enfants d'un endroit à l'autre (III, 86, p. 95).

Gervais n'est pas le seul à transmettre cette idée. Déjà attestée dans un décret de l'évêque de Worm et chez Gautier de Map au XII ${ }^{\mathrm{e}}$ siècle, elle réapparaît, sensiblement à la même époque (circa 1244), chez Vincent de Beauvais qui souligne lui aussi ce penchant vampirique des démons:

Nama mare daemones sanguinem dicuntur (Speculum doctrinale, IX, 120);

Decitur tamen diabolus se inserere sanguinibus, id est humoribus, quia in virtute imaginativam quae abundant humoribus, imprimit imagines rerum delectabilium, ex quibus surgunt malae cogitationes dum anima circa eas occupatur (Speculum naturale, II, 118) ${ }^{70}$.

Voici qui confirmerait la nature de l'épisode d'Avalon comme pure mirage ou pure fantasmagorie aux contours diaboliques fichée en plein univers poétique de la chanson de geste. Quant aux réminiscences vernaculaires du vampirisme, elles sont plutôt rares avant le XIII ${ }^{\mathrm{e}}$ siècle. Vers la fin du XII ${ }^{\mathrm{e}}$ siècle, nous en décelons néanmoins la trace dans Robert le diable, un roman anonyme en vers qui raconte les aventures d'un enfant conçu à la suite d'un pacte avec le démon et dont la trajectoire est constamment marquée par le sang: sang de toutes ses victimes innocentes violemment assassinées

\footnotetext{
${ }^{70}$ Apud ARAYRE, M. - «Le sang sans le Speculum Maius...», p. 349.
} 
auxquelles il perce le cœur (qui, outre ses connotations métaphysiques et poétiques, représente le lieu où le sang prend sa source selon la tradition médicale ${ }^{71}$ ) et sang nourricier qu'il préfère au lait. Là encore, Robert exhibe son désir de profanation et de destruction de l'ordre symbolique et culturel. Pour lui, en effet, le lait ne renvoie pas á ce sang impur blanchi (dont il pourrait ainsi s'alimenter), mais surtout à l'image (textuelle et iconographique) de la Vierge allaitant son enfant ${ }^{72}$ dont le anti-héros cherche délibérément à s'écarter ${ }^{73}$. Dans le contexte germanique, limitons-nous évoquer un roman arthurien post-classique, Daniel von dem blühenden Tal (circa 1220) ${ }^{74}$ qui met en scène la dimension polysémique du sang: on y découvre, en effet, à la fois le motif du sang rédempteur qui oblige le messager du diable, atteint d'une étrange maladie, à enlever (à travers le charme de sa voix) puis à tuer tous les hommes du pays pour se baigner dans leur sang (ce monstre sera tué par Daniel, modèle du héros libérateur et civilisateur) ${ }^{75}$, ainsi que le motif vampirique du sang nourricier, à travers l'histoire du monstre sans ventre (autre envoyé et compagnon du diable) qui, au moyen d'une tête de méduse, met à mort ses adversaires afin que lui et ses innombrables complices venus de l'indifférencié de la mer (trait également caractéristique des sarrasins dans la chanson de geste) viennent s'abreuver du sang de leurs victimes (cette fois-ci, à l'instar de son double mythique Persée, Daniel utilise un miroir pour éviter le regard pétrifiant de la méduse et tuer le montre).

\footnotetext{
${ }^{71}$ Vincent de Beauvais insiste avec force de redondance sur cet aspect qu'il considère essentiel: «Cor est in omnibus habentibus sanguinem. Vasa namque sanguinis venae sunt et harum principium est cor, unde venae videntur exire a corde, non transire per ipsum. Cor fons est et principium sanguinis. In corde est principium venarum et prima virtus creans sanguinis» (Speculum naturale, XXI, 26, apud TARAYRE, M. - «Le sang dans le Speculum Maius...», p. 347).

${ }^{72}$ Sur la spiritualisation allégorisante du corps et des fluides féminins à travers l'image du lait (lait de la Vierge, lait de la doctrine, etc.), je renvoie aux commentaires de SINCLAIR, F. E. - Milk and Blood, p. 17-51), L'HERMITE-LECLERCQ, P. - «Le lait et le sang de la Vierge», in Le Sang au Moyen Âge, p. 145-162), ainsi qu’á ceux de PERROT, J.-P. - «Du sang au lait...».

${ }^{73}$ GAUCHER, E. - «Sang vermeil, merveille du sen: à propos de Robert le Diable», in Le Sang au Moyen Âge, p. 217-226).

${ }^{74}$ Texte cité et commenté par BUSCHINGER, D. - «Sang versé, sang guérisseur, sang aliment, sang du Christ dans la littérature médiévale allemande», in Le sang au Moyen Âge, p. 257-266.

${ }^{75}$ Le motif du bain de sang qui guérit de la maladie (de la lèpre, notamment) est très répandu au Moyen Âge: nous le trouvons, par exemple, dans La Queste del saint Graal (la pucelle vierge qui donne son sang pour sauver le lépreux) et dans la chanson de geste Ami et Amile où c'est l'enfant qui est sacrifié pour sauver l'ami. Sur ce sujet, voir, entre autres, BERTHELOT, A. - «Sang et lèpre, sang et feu», in Le Sang au Moyen Âge, p. 39-68) et MCCRACKEN, P. -The Curse of Eve, p. 2-6; 41-60.
} 
$\mathrm{Au}$ spectre démoniaque du vampirisme, La Bataille Loquifer ajoute cependant une nouvelle couche mythique: le motif du talon comme point vulnérable du héros aux pouvoirs surnaturels. Un dernier texte, contemporain du précédant et appartenant à une même aire géographique et culturelle, nous permet de faire le lien entre les deux motifs apparemment antithétiques, celui du sang réparateur/protecteur et celui du corps d'où émerge la faille: il s'agit de La Chanson des Nibelungen ${ }^{76}$ qui s'ouvre avec le départ de Siegfried pour épouser Kriemhild. Comme au début de la légende de Tristan, le héros tue sur son chemin un dragon et, suivant une coutume rituelle vraisemblablement bien établie, il se baigne dans son sang afin de devenir invulnérable. Cependant, une feuille de tilleul se pose entre les omoplates du héros, empêchant le sang de couler à cet endroit. Siegfried, tout comme Achille dans la tradition grecque, révèle donc un point faible et, indépendamment des maillons de la tradition qui ont fourni à La Bataille Loquifer ce motif ${ }^{77}$, Rainouart est, à n'en point douter, héritier et successeur de cette lignée de héros mythiques appartenant au panthéon indo-européen ${ }^{78}$.

\footnotetext{
${ }^{76}$ Trad. du moyen-haut-allemand par BUSCHINGER, D. et PASTRE, J.-M. Paris : Gallimard, 2001.

${ }^{77}$ Nous retrouvons ce motif dans un roman presque contemporain de la Bataille, Le Haut Livre du Graal (Perlesvaus) où Gauvain tue, durant son parcours tumultueux, un descendant du lignage d'Achille en lui enfonçant justement l'épée dans la plante du pied (Branche V, p. 299 de l'éd. de STRUBEL, A. Paris : Librairie Générale Française, coll. «Lettres Gothiques», 2007). L'éditeur reconnaît ici l'étrangeté de cette allusion déplacée dans le contexte qu'il fait remonter à une légende tardive transmisse en particulier par Fulgence. La Bataille Loquifer aurait-elle bu à la même source textuelle que le Perlesvaus ou, là encore, comme pour le personnage de Chapalu, aurait-elle puisé dans une source orale? La réponse est délicate, mais le fait est que, du moins à ma connaissance, aucun texte vernaculaire appartenant à la dénommé «matière antique» (Roman de Thèbes, Énéas, Roman de Troie) ne fait référence à la mort d'Achille provoquée par une flèche décochée par Pâris (instigué par Apollon) qui atteint son point vulnérable, le talon. Cette version, assez tardive dans la tradition manuscrite, n'est d'ailleurs transmise pour la première fois que par Stace (poète du I ${ }^{\text {er }}$ siècle après J.-C.) dans son Achilléide (où l'on peut lire également l'origine de ce point faible: ayant été baigné dans les eaux infernales du Styx par Thétis, le corps d'Achille est invulnérable à l'exception du talon par lequel sa mère l'avait tenu). Cela n'empêche, comme en témoignent certaines représentations iconographiques trouvées sur des vases de la période archaïque et du début de la période classique, que la tradition du «talon d'Achille» fut très ancienne. Sur cette tradition, voir GANTZ, T. Timothy - Early Greek Myth. Baltimore: Johns Hopkins University Press, 1993.

${ }^{78}$ Voir, à ce propos, PASTRE, J.-M. - «Rainouart et Rennewart: un guerrier aux cuisines», in GUIDOT, B. - Burlesque et dérision dans les épopées de l'occident médiéval (Actes du Colloque International des Rencontres Européennes de Strasbourg). Paris: Les Belles Lettres, coll. Littéraires, 3, p. 123-131; MARTIN, J.-P. - «Le personnage de Rainouart, entre épopée et carnaval», in Comprendre et aimer la chanson de geste (À propos d'Aliscans). Fontenay aux Roses : Feuillets de l'E. N. S. Fontenay-St. Cloud, 1994, p. 63-86.
} 
Quelles conclusions tirer de cette vaste constellation mythique, mythologique théologique et littéraire qui entoure l'épreuve de sang à laquelle Rainouart doit se soumettre à son arrivée à Avalon? Que cet univers représente le monde des morts - le monde de la mort - nul n'en doute. Comme dans le Château des Reines Mortes que Gauvain rencontre dans la seconde partie du Conte du Graal de Chrétien de Troyes, les fonctions sociales y sont bloquées, le temps s'y trouve aboli et diverses générations s'y côtoient $^{79}$ : Brunehot aux côtés de sa file Morgue, Arthur auprès de sa mère Ygerne qui se repose sous un «pin ramé» (v. 3906), symbole bien connu de l'immortalité. Nul ne doute non plus que Chapalu et les fées (qui s'emparent du corps et de l'âme du héros durant son sommeil) s'apparentent aux striges décrites par Gervais de Tilbury ou aux âmes de tous ces défunts qui, selon une croyance quasi universelle, hantent le monde des vivants en quête de sang vif et chaud ${ }^{80}$. Remarquons d'ailleurs, sur le plan strictement littéraire, que tous les héros épiques et romanesques à l'exception d'Arthur (i.e., Perceval, Yvain, Gauvain et Roland) apparaissent comme des personnages complètement statiques qui ne jouent aucun rôle dans cet Autre-Monde de pacotille, qui ne prennent jamais la parole. Ce sont donc de pures formes-types, fruits d'une rhétorique épuisée et gaste, vidée de son sens et coupée de son rapport à une matière originelle déjà sentie, sans doute, comme trop lointaine. Désignés à peine par un signifiant nominal, ces figures ne sont qu'une présence reliquaire de la tradition poétique. Il incombera donc à Rainouart d'ébranler ces formes figées dans le lieucommun de la topique littéraire, et, par l'oblation de son sang, de redonner vie et forme humaine à ces lettres mortes de la fiction romanesque et épique. En instaurant une «écriture nouvelle» ${ }^{81}$, le sang devient clairement, dans ce contexte, emblème d'une rhétorique placée sous le signe de la rejuvenatio telle que l'a définie, sensiblement à la même époque, Geoffroi de Vinsauf dans sa Poetria nova (v. 1142 sq.):

\footnotetext{
${ }^{79}$ Ce qui confirmerait également cette étrange ressemblance entre Rainouart et Perceval, les deux héros étant, chacun à leur façon, sommé de répondre aux énigmes teintées de sang (Graal eucharistique, Lancequi-Saigne, plaies symboliques) qui émanent de cet Autre-Monde (celui du Roi Pêcheur ou celui de Morgue) paralysé par la blessure et la mort.

${ }^{80}$ Voir ROUX, J.-P. - Le Sang..., p. 221-234.

${ }^{81}$ Comme le suggérait également A. LEUPIN à propos de Raoul de Cambrai (voir passage cité en exergue).
} 
Ans qu'il eüst a la terre versé [Rainouart]

l'a Chapalus si forment agrapé

que li ronpit la chause et lou souler

et del talon a lopu cuir reversé;

li sans en est tout contreval filé;

cil s'abaissa qui molt l'a desiré,

si en ait bu, et sucié, et lapé.

Es vos son cors changié et remüé;

an forme d'ome est li chas tremüé.

Lou poil ot blont, menu recercelé,

les iolz ot vars et lou vis coloré,

gros fut par pis, graille par lou baldré (v. 3858-69).

Contrairement au duel contre Ysabras ou Loquifer, le combat contre Chapalu n'a ni vainqueur, ni vaincu. Plongé dans une atmosphère fortement mythique et symbolique, ce corps à corps ne participe plus d'une logique strictement narrative. Le rituel de sang qui rétablit l'ordre symbolique projeté sur l'image de ce corps redevenu modèle de beauté et d'harmonie, dissimulerait-t-il alors une sorte de pacte célébrant la nouvelle alliance entre la geste et le roman? Réponse hasardeuse, comme il arrive avec la plupart des questions soulevées par ce récit. Car, si pacte il y a, il s'agit d'un pacte bien fragile et éphémère qui n'annule pas pour autant toutes les tensions et contradictions entre les deux univers en présence. Que penser, par exemple, de la figure de Morgue qui, enceinte de Corbon, encore «.I. vif diable» (v. 3923), teinte soudain - et de façon tout à fait inédite - son discours d'une coloration typiquement épique et féodale centrée sur la biopolitique du lignage, attitude qui motivera d'ailleurs son projet vengeur de faire périr le héros afin que celui-ci ne retrouve Maillefer et que Corbon puisse hériter des riches fiefs de Porpaillart et de Toulouse ${ }^{82}$ D'autre part, remarquons que, malgré la promesse qu'il fait à son bienfaiteur de le servir fidèlement et de le conduire auprès de son fils (v.

\footnotetext{
${ }^{82}$ Après Rainouart lui avoir appris son désir de quitter Avalon pour partir en quête de Maillefer, Morgue, la fée-amante et source de vie, assume à nouveau son visage destructeur et mortel: «Morgue l'antent, lou sanc cuide desver./ Chapalu fait isnelement mander:/ 'Ami,' dist elle, 'ses que voil conmander?/ Tu conduiras Renoart par la mer,/ et se il puet Maillefer conquester,/ Corbans mes fils ne poroit rien clamer/ a Porpaillart, n'a Tolose sor Mer'» (3949-55).
} 
3871-3880), l'ingrat Chapalu - qui n'a fait que dissimuler l'amertume qu'il ressent à l'égard de Rainouart à la suite des coups reçus lors du duel et qui demeure en fait entièrement prisonnier des sortilèges et des lois d'Avalon dictées par Morgue - ne manquera pas de trahir le héros la première occasion venue en faisant naufrager son navire en pleine mer (laisse 92). Ainsi, en dispersant les enchantements du Royaume de Féerie, Rainouart délivre et régénère, certes, par le don de son sang, la matière arthurienne prisonnière du carcan d'une topique romanesque qu'elle a elle-même créé. Vaincre le monstre et quitter sans entraves l'Autre-Monde de la mort témoignerait ainsi de la supériorité de la geste sur la matière arthurienne ${ }^{83}$. Cependant, la présence de Roland (et non celle de Charlemagne, ce qui compromettrait le devenir de la Geste du Roi) aux côtés d'Arthur, révèle que le geste du héros sauve également l'épopée carolingienne traditionnelle aux prises, elle aussi, avec un modèle narratif, idéologique et imaginaire apparemment épuisé, inopérant et sans doute inadéquat pour répondre aux transformations profondes subies par la civilisation médiévale. L'épisode du naufrage au cours duquel Rainouart, le corps transpercé et déchiré par les épaves, se trouve au seuil de la mort, est à cet égard très révélateur. L'heure est à la confession, au planctus et à la prière. Dans son credo épique peu orthodoxe, le héros invoque la sainte Vierge, la belle sirène qu'il avait accepté de libérer peu de temps auparavant (laisse 87) et saint Julien (laisse 93). Cependant, comme si toute sémiologie épique était vouée à l'échec, aucun des adjuvants célestes ne viennent en aide à Rainouart, et ce sera à nouveau un être extérieur à cet univers poétique, un être une fois encore lié à une image mythique et ambivalente de la féminité (la sirène reconnaissante ${ }^{84}$ ), que nous devrons la survie de ce nouvel Ulysse et celle de la geste.

Rainouart se réveille alors là où il s'était endormi. La circularité même de l'épisode, qui commence et s'achève sur les rives de Porpaillart ${ }^{85}$, confère au séjour à Avalon

\footnotetext{
${ }^{83}$ Voir, à ce sujet, ANDRIEUX, N. - «Arthur et Charlemagne réunis en Avalon...», p. 433.

${ }^{84}$ Cette reconnaissance constitue l'exacte envers de la monstruosité résultant de la transgression de l'interdit sur le corps féminin (Ysabras et Chapalu).

${ }^{85}$ Le passage s'ouvre et se clôt sur un vers pratiquement identique: «Renoars iert sor mer en .I. laris» (v. 3603); «Renoars dort sor mer an .I. larris» (v. 4211).
} 
l'inconsistance d'un rêve, d'une irréalité. Récit hors-texte, palimpseste épique ${ }^{86}$ suspendu dans le temps et destiné à l'effacement, La Bataille Loquifer se clôt sur le retour du héros à Porpaillard, oublieux de tout, même de la quête de son fils qui avait pourtant motivé son départ. L'extrême ambiguïté sur laquelle se clôt ce poème soulève ainsi bien plus de questions qu'elle n'offre de réponses. À une époque où le chant épique est concurrencé et menacé par la multiplication des cycles romanesques, ce rapport dialogique entre les genres n'est pas exempt de tensions qui relèvent à la fois de la complicité inavouable et secrète, de la rivalité latente, du conflit déclaré ou de la complémentarité. Ainsi, face à une descendance diabolique (Corbon) issue de l'union apparemment contre-nature de l'épopée et du roman (de Rainouart et de Morgue), face, d'autre part, à la trahison perpétrée par le conte de Bretagne (en la personne de Chapalu) qui, en tuant le héros, compromet sérieusement le devenir de la geste carolingienne, cherchant même à s'emparer de son héritage, à usurper son territoire fictionnelle, il reviendra à Picolet (personnage éminemment arthurien) de défendre, par le biais de Maillefer, le lignage de Rainouart, de garantir son droit légitime au patrimoine matériel et poétique de l'univers carolingien, d'assurer, en un mot, la survie d'une chanson de geste nécessairement enrichie par le contact avec cette matière radicalement autre que le nain $^{87}$ et Avalon représentent. Si, par la façon dont elle bouleverse les structures idéologiques et poétiques de la chanson de geste traditionnelle et du roman, La Bataille Loquifer marque simultanément la fin d'une écriture et l'accomplissement d'une parole, comme le faisait remarquer Nelly Andrieux ${ }^{88}$, elle inaugure également, dans cette perspective, un nouveau langage du et sur le sang. Ce sang qui pénètre, purifie et féconde la terre usurpée ou gaste, qui imprègne l'armure et la peau des guerriers, qui régénère par le sacrifice rédempteur, dont on cherche à stopper l'hémorragie ou à fuir les effets néfastes ou parfois même toxiques, qui s'érigent en grammaire du lignage dont dépend la place du sujet dans la vaste syntaxe de l'univers et de la société, qui, à la fin du récit, alimente et transforme l'autre, ce sang n'est plus, dans La Bataille Loquifer, un symbole transparent et pré-codifié. Au sein d'un univers social et idéologique en

\footnotetext{
${ }^{86}$ Pour reprendre le beau titre des études recueillies par BOUTET, D. et ESMEIN-SARRASIN, C. Palimpsestes épiques. Récritures et interférences génériques. Paris: Presses de l'Université ParisSorbonne, 2006.

${ }^{87}$ Dans sa qualité de protecteur du lignage épique, Picolet joue ici un rôle analogue à celui d'Aubéron dans Huon de Bordeaux.

${ }^{88}$ «Arthur et Charlemagne réunis en Avalon...», p. 433.
} 
pleine mutation, l'image du sang de Rainouart coulant désormais dans les veines de Chapalu est devenu signe poétique paradoxal au cœur d'un récit non moins ambivalent qui s'érige en métaphore exemplaire de cette admirable bâtardise de l'écriture qui gouverne le discours épique à partir du XIII ${ }^{\mathrm{e}}$ siècle, un discours placé sous le signe de la confluence, du mélange des genres et de la rencontre - parfois tendue et au dénouement toujours incertain - entre le mythe, la fiction et l'idéologie. Plus que jamais, le sang se transforme en hypostase du sens dont la fluidité lui permet d'en épouser la trajectoire et les formes symboliques en se répandant sur cette «erbe [...] roge, tainte et encoloree» (v. 2268) de ce champ de bataille d'où retentissent, de plus en plus fort, les nouveaux enjeux du chant épique.

La fu l'estors et li chans afinés, et la bataille et li chans jurés (v. 1291-92).

\section{Bibliographie:}

ALISCANS. Ed. Cl. Régnier. Paris : Champion, 2 vols, 1990.

BATAILlE LOQUIFER (LA). Éd. M. Barrett. Meddium Aevum Monographs, New Series, 6: Oxford, Society for the Study of Mediaeval Languages and Literatures, 1975.

BERTHELOT, A., «Sang et lèpre, sang et feu», in Le Sang au Moyen Âge, op. cit.p. 39$68)$.

BLOCH, H., Étymologie et généalogie. Une anthropologie littéraire du Moyen Âge français. Paris: Seuil, 1989.

BOUTET, D.; ESMEIN-SARRASIN, C., Palimpsestes épiques. Récritures et interférences génériques. Paris : Presses de l’Université Paris-Sorbonne, 2006. 
BUSCHINGER, D., «Sang versé, sang guérisseur, sang aliment, sang du Christ dans la littérature médiévale allemande», in Le sang au Moyen Âge, op. cit., p. 257-266.

CHANSON DE ROLAND (LA). Ed. I. Short. Paris : Librairie Générale Française, Coll. Lettres Gothiques, 1990).

CHANSON DES NIBELUNGEN (LA). Tradução de D. BUSCHINGER E J.-M. PASTRE. Paris : Gallimard, 2001.

CHRETIEN DE TROYES, Le Chevalier de la Charrete (Lancelot). Ed. M. Roques. Paris : Champion, 1983.

CLAMOTE CARRETO, Carlos F., «Rainouart au pays des fées. Interchangeabilité des personnages et dialogisme dans "La Bataille Loquifer"», in CONNNOCHIEBOURGNE, Ch. (dir.), Façonner son personnage au Moyen Âge. Senefiance, 53. Aixen-Provence : PUP, 2007, p. 99-122.

GANTZ, T. T., Early Greek Myth. Baltimore: Johns Hopkins University Press, 1993.

GAUCHER, E., «Sang vermeil, merveille du sen: à propos de Robert le Diable», in Le Sang au Moyen Âge, op. cit., p. 217-226.

GERVAIS DE TILBURY, Livre des Merveilles (Otia Imperalia, III, 86). Tradução e notas de A. DUCHESNE. Paris : Les Belles Lettres, 2004.

LE HAUT LIVRE DU GRAAL (PERLESVAUS). Ed. A. Strubel. Paris : Librairie Générale Française, coll. «Lettres Gothiques», 2007

HUCHET, J.-Ch., Tristan et le sang de l'écriture. Paris : PUF, 1990.

ISNART, C., «La fée, la grotte et le tisserand. Etude ethnologique autour de la Monographie communale d'Utelle [circa 1910]». Pays Vésuvien, 1 (2000), p. 147-157.

JACQUARD. D.; THOMASSET, Cl. - Sexualité et savoir médical au Moyen Âge. Paris : PUF, 1985. 
JAMESON, F., The Political Unconscious: Narrative as a Socially Symbolic Act. New York, Ithaca: Cornell University Press, 1981.

KAY, S., The Chanson de Geste in the Age of Romance. Political Fictions. Oxford: Clarendon Press, 1995.

LACROIX, D. ; WALTER, Ph., Tristan et Iseut: les poèmes français, la saga norroise. Paris : Librairie Générale Française, Coll. Lettres Gothiques, 1989

LE GOFF, J. ; TRUONG, N., Une histoire du corps au Moyen Âge. Paris : Liana Levi, 2003.

LE GOFF, J., «Métiers licites et métiers illicites dans l'Occident médiéval», in Pour une autre Moyen Âge. Temps, travail et culture en Occident. Paris : Gallimard, 1977, p. 91107.

LEUPIN, A., «Raoul de Cambrai: la bâtardise de l'écriture», Romanic Review, 79 (1988), p. 89-104.

Fiction et Incarnation. Littérature et théologie au Moyen Age. Paris: Flammarion, 1993.

LOTÁRIO DE SEGNI, De miseria humanae condicionis. Ed. R. E. Lewis : Atenas, 1978.

MARTIN, J.-P. - «Le personnage de Rainouart, entre épopée et carnaval», in Comprendre et aimer la chanson de geste (À propos d'Aliscans). Fontenay aux Roses : Feuillets de l'E. N. S. Fontenay-St. Cloud, 1994, p. 63-86.

MCCRACKEN, P., The Curse of Eve, The Wound of the Hero. Blood, Gender, and Medieval Literature. Philadelphia: University of Pennsylvania Press, 2003.

MOniage RaInouart (LE) I. Ed. G. A. Bertin. Paris : Éd. A. \& J. Picard \& C , $^{\text {, }}$ 1973. 
ANDRIEUX, N., «Arthur et Charlemagne réunis en Avalon: la Bataille Loquifer ou l'accomplissement d'une parole», in Actes du IX Congrès International de la Société Rencesvals. Modena : Mucchi, vol. 2, 1984, p. 425-434.

OLLIER, M.-L., La Forme du sens. Textes narratifs des $\mathrm{XII}^{\mathrm{e}}$ et $\mathrm{XIII}^{\mathrm{e}}$ siècles. Études littéraires et linguistiques. Orléans : Ed. Paradigme, 2000.

PALAZZO, E. - Liturgie et société au Moyen Âge. Paris: Aubier, 2000.

PASTRE, J.-M., «Rainouart et Rennewart: un guerrier aux cuisines», in GUIDOT, B. Burlesque et dérision dans les épopées de l'occident médiéval (Actes du Colloque International des Rencontres Européennes de Strasbourg). Paris : Les Belles Lettres, coll. Littéraires, 3, p. 123-131.

PAYEN, J.-Ch., «Une poétique du génocide joyeux: de voir de violence et plaisir de tuer dans la Chanson de Roland», Olifant, 6, 3-4 (1979), p. 226-236.

PERROT, J.-P., «Du sang au lait: l'imaginaire du sang et ses logiques dans les Passions de martyrs», in Le Sang au Moyen Âge, op. cit., p. 459-470.

PLACIDES ET TIMEO OU LI SECRES AS PHILOSOPHES, Ed. crítica de Cl. Thomasset. Genève : Droz, 1980.

ROUSSEL, Cl., «Le mélange des genres dans les chansons de geste tardives», in ALVAR, C. e PAREDES, J. (ed.), Les Chansons de geste. Actes du XVI ${ }^{\mathrm{e}}$ Congrès Internationale de la Société Rencesvals pour l'Étude des Épopées Romanes (Granada, 21-25 juillet 2003). Granada : Université de Granada, 2005, p. 64-85.

ROUX, J.-P., Le Sang. Mythes, symboles et réalités. Paris : Fayard, 1988.

RUBIN, M., Corpus Christi: The Eucharist in the Late Medieval Culture. Cambridge: Cambridge University Press, 1991.

SINCLAIR, F. E., Milk and Blood. Gender and Genealogy in the «Chanson de Geste». Berne: Peter Lang, 2003. 
STANESCO, M., «Du démon de midi à l'Éros mélancolique: topologie du féerique dans le lai narratif breton». Poétique, 106 (1996), p. 131-159.

TARAYRE, M., «Le sang dans le Speculum Maius de Vincent de Beauvais. De la science aux miracula», in FAURE, M. (ed.) - Le Sang au Moyen Âge. Actes du quatrième colloque international de Montpellier, 27-29 novembre 1997). Les Cahiers du C.R.I.S.I.M.A., 4. Montpellier : Presses de l’Université Paul Valéry, 1999, p. 343359.

THOMASSET, Cl., «De la nature féminine», in KLAPISCH-ZUBEL, Ch. (dir.) Histoire des femmes en Occident. II - Le Moyen Âge. Paris : Plon, 2002, p. 65-98.

TRACHSLER, R., Disjointures - Conjointures. Étude sur l'interférence des matières narratives dans la littérature française du Moyen Âge. Tübingen-Basel: A. Francke Verlag, Romanica Helvetica, 2000.

VAN PROYEN, M., «Sang et hérédité. À la croisée des imaginaires médicaux et sociaux des XIII ${ }^{\mathrm{e}}$ et XIV ${ }^{\mathrm{e}}$ siècles», in Le sang au Moyen Âge, op. cit., p. 69-75.

VINCENSINI, J.-J., «Viol de la fée, violence du féerique. Remarques sur la vocation anthropologique de la littérature médiévale». Senefiance, 36 (1994), p. 545-559.

VOISENET, J., «Le tabou du sang dans les pénitentiels du haut Moyen Âge», in Le Sang au Moyen Âge, op. cit., p. 111-125. 


\section{COMO CITAR ESTE ARTIGO}

\section{Referência electrónica:}

CARRETO, Carlos Clamote F. - “«Li sens conmence contreval a filer...». Imaginaire du sang et hétérodoxies épiques dans La Bataille Loquifer”. Medievalista [Em linha]. No 10, (Julho de 2011). [Consultado dd.mm.aaaa]. Disponível em http://www2.fcsh.unl.pt/iem/medievalista/MEDIEVALISTA10lcarreto1005.html. ISSN 1646-740X.

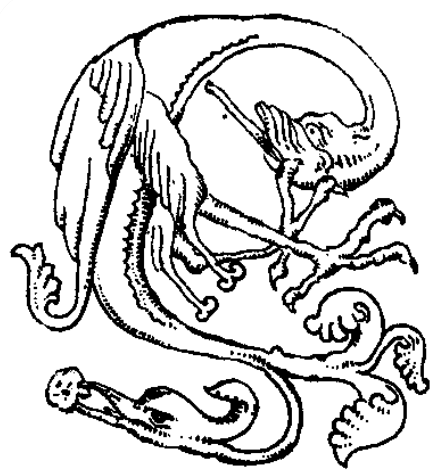

